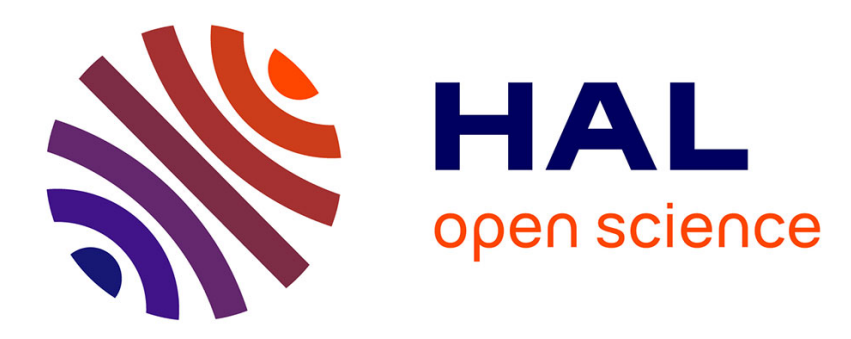

\title{
Homogenization of cellular sandwich panels
}

\author{
Arthur Lebée, Karam Sab
}

\section{To cite this version:}

Arthur Lebée, Karam Sab. Homogenization of cellular sandwich panels. Comptes Rendus Mécanique, 2012, 340 (4-5), pp.320-337. 10.1016/j.crme.2012.02.014 . hal-00794221

\section{HAL Id: hal-00794221 \\ https://hal.science/hal-00794221}

Submitted on 25 Feb 2013

HAL is a multi-disciplinary open access archive for the deposit and dissemination of scientific research documents, whether they are published or not. The documents may come from teaching and research institutions in France or abroad, or from public or private research centers.
L'archive ouverte pluridisciplinaire HAL, est destinée au dépôt et à la diffusion de documents scientifiques de niveau recherche, publiés ou non, émanant des établissements d'enseignement et de recherche français ou étrangers, des laboratoires publics ou privés. 


\title{
Homogenization of cellular sandwich panels
}

\author{
Arthur Lebée ${ }^{\mathrm{a}}$, Karam Sab*a \\ ${ }^{a}$ Université Paris-Est, Laboratoire Navier (École des Ponts ParisTech, IFSTTAR, CNRS). \\ École des Ponts ParisTech, 6 et 8 avenue Blaise Pascal. \\ 77455 Marne-la-Vallée cedex2 \\ tel. +33-1-64153749, fax. +33-1-64153741 \\ Received $* * * * *$; accepted after revision +++++ \\ Presented by
}

\begin{abstract}
The Bending-Gradient plate theory originally presented in [1] is applied to cellular sandwich panels. This theory is the extension of Reissner-Mindlin theory to heterogeneous plates. Its application clarifies common assumptions made in sandwich theory. It also enables to define a direct homogenization scheme for deriving the shear forces stiffness of sandwich panels. Finally, the conventional bounds used for estimating sandwich panels stiffnesses are justified. To cite this article: Arthur Lebée, Karam Sab, C. R. Mecanique?? (201?).
\end{abstract}

Key words: Plate theory; Sandwich panels ; Homogenization ; Periodic plates ; Higher-order models ; Periodic plates

\section{Introduction}

Sandwich panels are well known for their structural efficiency. They are made of a light and thick core which is glued between two stiff skins. Usually the skins are quite dense and made of material with high mechanical properties whereas the core is lighter and achieves a compromise between weight and mechanical properties. Many types of core are currently used and the field is still innovative today. One can distinguish two main categories: homogeneous cores (including foams) and cellular cores such as honeycomb, truss cores [2] and folded cores [3]. It is commonly accepted that mainly the skins contribute to the flexural stiffness and that the core mostly affects the shear forces stiffness of the sandwich panel. This is because the skins are put under traction and compression and the core is not really loaded when bending the sandwich panel; whereas most of the transverse shear related to shear forces is carried by the core since the skins are thin. In this very simple analysis, two assumptions lie: a contrast assumption (the

Email addresses: arthur.lebee@enpc.fr (Arthur Lebée), karam.sab@enpc.fr (Karam Sab*). 
skins are "much stiffer" than the core) and a "thin skins" assumption. It turns out that going further than this very intuitive description raised a certain amount of difficulties especially regarding the transverse shear behavior of cellular sandwich panels. Though, the shear deflection cannot be neglected in sandwich panel design because the core is rather soft and an accurate knowledge of the shear forces stiffness is necessary.

Thorough reviews of the mechanical behavior of sandwich panels already exist $[4,5,6]$. A large variety of models was suggested in order to provide satisfying description of their complex behavior. The simplest one is Reissner-Mindlin model [7, 8] which simply takes into account deflection related to transverse shear. This model requires the derivation of the shear forces stiffness tensor ${ }^{1} \underset{\sim}{\boldsymbol{F}}=\left(F_{\alpha \beta}\right)$ which links the shear forces $\underline{\boldsymbol{Q}}=\left(Q_{\alpha}\right)$ to the shear strain $\underline{\boldsymbol{\gamma}}=\left(\gamma_{\alpha}\right)$ through the constitutive equation: $\underline{\boldsymbol{Q}}=\underset{\sim}{\boldsymbol{F}} \cdot \underline{\boldsymbol{\gamma}}$. Because the derivation of the shear forces stiffness tensor in terms of the local constitutive elastic properties is not fully consensual, Reissner-Mindlin model applied to sandwich panels is not completely accepted. Recently Lebée and Sab [1] and [9] derived a new plate model, the Bending-Gradient plate model. It is the extension of the classical Reissner-Mindlin model to heterogeneous plates. In this paper, we apply the Bending-Gradient plate theory to a sandwich panel in order to put into perspective previous work and provide efficient tools for estimating the shear forces stiffness of cellular sandwich panels.

The paper is organized as follows. First, a non-exhaustive bibliography is provided in order to point out several difficulties encountered when deriving sandwich panels shear forces stiffness (Section 2). Then, the Bending-Gradient plate theory and its homogenization scheme are briefly summarized in Section 3 . This scheme is separated in two steps. First the Kirchhoff-Love stiffnesses are derived and then the transverse shear behavior of the plate is determined. Thus, in Section 4, the in-plane behavior of sandwich panels is derived and the contrast assumption is introduced. Then, in Section 5, the application of the transverse shear auxiliary problem reveals that the actual shear behavior of a sandwich panel under the contrast assumption is a Reissner-Mindlin one. This enables the justification of already known bounds for the shear forces stiffness. Finally, a discussion on the assumptions commonly made with sandwich panels is provided in Section 6.

\section{Questions raised by sandwich panels shear forces stiffness}

We consider sandwich panels made of two identical and homogeneous skins of thickness $t_{s}$ and a core of thickness $t_{c}$ which can be either homogeneous or periodically heterogeneous (Figure 1). The overall thickness of the sandwich panel is $h=t_{c}+2 t_{s}$. The present analysis is done in the framework of linearized elasticity. The sandwich panel reference frame is $\left(\underline{\hat{e}}_{1}, \underline{\hat{e}}_{2}, \underline{\hat{e}}_{3}\right)$ where $\underline{\hat{e}}_{3}$ is the out-of-plane direction and $\underline{\hat{\boldsymbol{y}}}=\left(y_{1}, y_{2}, y_{3}\right)$ denotes the local coordinates of the unit-cell. The skins $3 \mathrm{D}$ elasticity stiffness tensor is $\stackrel{\hat{\approx}}{ }^{s}=\left(C_{i j k l}^{s}\right)$ and the core stiffness is $\hat{\boldsymbol{C}}^{c}(\underline{\hat{\boldsymbol{y}}})$. We assume also that the skins and the core constitutive material (when the latter is homogeneous) follows a local symmetry with respect to $\left(y_{1}, y_{2}\right)$ plane $\left(C_{\alpha 333}=\right.$ $C_{\alpha \beta \gamma 3}=0$, monoclinic symmetry). The transverse shear stiffness tensor writes then as: $\boldsymbol{G}^{s}=\left(G_{\alpha \beta}^{s}\right)=$ $\left(C_{\alpha 3 \beta 3}^{s}\right)$ and $\boldsymbol{G}^{c}=\left(G_{\alpha \beta}^{c}\right)=\left(C_{\alpha 3 \beta 3}^{c}\right)$.

Two additional assumptions are commonly made when considering sandwich panels. First, a contrast assumption enables to neglect the contribution of the core to the bending stiffness. It is often formulated as: $t_{c} G^{c} \ll t_{s} E^{s}$ ( $E$ denotes Young modulus) in the case of homogeneous core and isotropic materials

1. Greek indices $\alpha, \beta, \gamma=1,2$ denote $2 \mathrm{D}$ tensors and Latin indices $i, j, k=1,2,3$ denote $3 \mathrm{D}$ tensors. Einstein convention on indices summation is used. When tensor notation is used, the character is bold and $\hat{\bullet}$ is added when it is a $3 \mathrm{D}$ tensor otherwise it is a $2 \mathrm{D}$ tensor. 


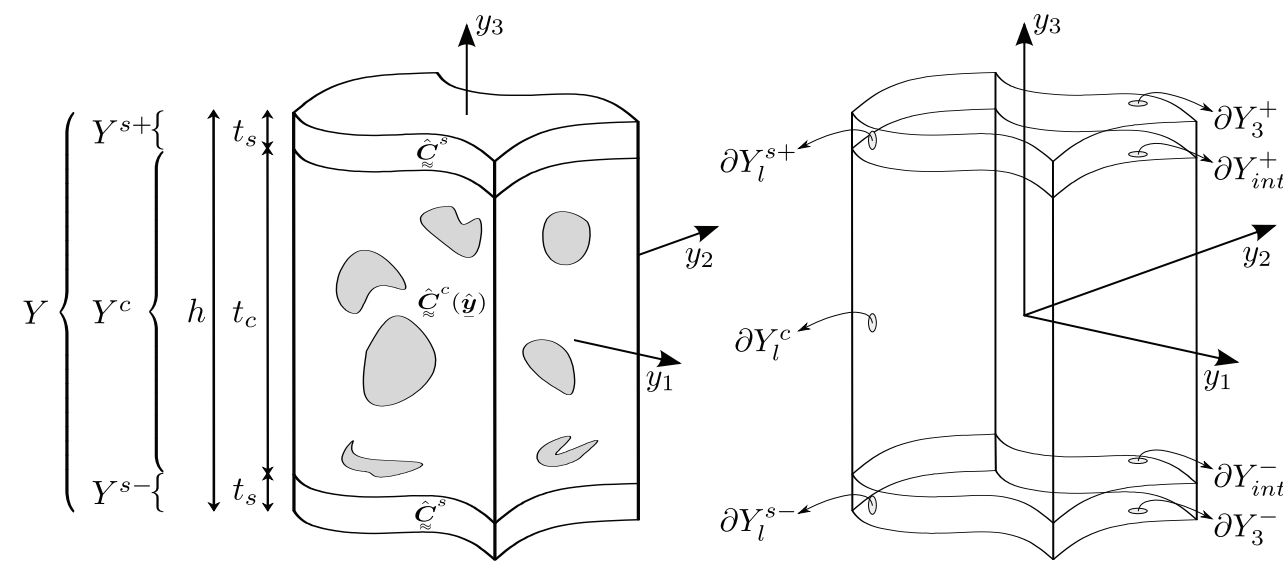

Figure 1. Cellular sandwich panel unit-cell

(i.e. $G_{\alpha \beta}^{c}=G^{c} \delta_{\alpha \beta}$ where $\delta_{\alpha \beta}$ is Kronecker's symbol). The second assumption is that the thickness of the skins is very small with respect to the thickness of the core so that the contribution of the skins to the overall shear forces stiffness can be neglected: $t_{s} \ll t_{c}$. Actually, this may be antagonistic with the contrast assumption and many authors tried to relax this assumption as will be illustrated in the following.

\subsection{The case of homogeneous cores}

The concept of sandwich panel is rather old but most of the theoretical work started with the sandwich panel including balsa core used in the De Havilland Mosquito. Several contributions emerged just after the second world war (see [4] for details) but the simplest suggestion came from Reissner [10] for isotropic sandwich panel who assumed both $t_{c} G^{c} \ll t_{s} E^{s}$ and $t_{s} \ll t_{c}$ :

$$
F_{\alpha \beta}=t_{c} G^{c} \delta_{\alpha \beta}
$$

Later, Reissner [11] assumed uniform shear strain in the core and rigid skins. He also chose $a d$ hoc bounds for integration through the thickness and obtained:

$$
\underset{\sim}{\boldsymbol{F}}=\left(t_{c}+t_{s}\right){\underset{\sim}{\boldsymbol{G}}}^{c}=\left(1+\frac{t_{s}}{t_{c}}\right) t_{c} \boldsymbol{G}^{c}
$$

This formula is still used in practice today [12]. Allen [4] based on previous work used the same kinematic assumptions without changing the integration through the thickness and suggested:

$$
\underset{\sim}{\boldsymbol{F}}=\frac{\left(t_{c}+t_{s}\right)^{2}}{t_{c}} \boldsymbol{G}^{c} \approx\left(1+2 \frac{t_{s}}{t_{c}}\right) t_{c}{\underset{\sim}{ }}^{c}
$$

Additionally, Kelsey et al. [13] suggested, with neither justification nor reference found by the authors, the following shear forces stiffness:

$$
\underset{\sim}{\boldsymbol{F}}=\frac{t_{c}}{k_{s}^{2}} \boldsymbol{G}^{c} \approx\left(1+2 \frac{t_{s}}{t_{c}}\right) t_{c} \boldsymbol{G}^{c}, \quad \text { where } \quad k_{s}=\frac{3}{2} \frac{t_{c}\left(h^{2}-t_{c}^{2}\right)}{h^{3}-t_{c}^{3}}
$$

All these suggestions can be written as $\underset{\sim}{\boldsymbol{F}}=A_{S} \boldsymbol{G}^{c}$ where $A_{S}$ is similar to the shear area for beams. When $t_{s}=0, A_{S}=t_{c}$ in all cases. It seems that when $t_{s}$ is not negligible the authors tried to take into account the larger "lever effect" of the skins on the core (the centroids distance between skins is $t_{c}+t_{s}$ ). 
However, the formulas are not fully consistent at first order.

Since only $\boldsymbol{G}^{c}$ is involved in these formulas, the denomination "transverse shear stiffness" for $\underset{\sim}{\boldsymbol{F}}$ is rather common with sandwich panels. Because sandwich panels are heterogeneous, this simplification is actually meaningless and even sometimes misleading. In the present work, a clear distinction is made between the transverse shear stiffness $\boldsymbol{G}$ which refers to a local property of the constitutive material and the shear forces stiffness $\underset{\sim}{\boldsymbol{F}}$ which refers to the structural stiffness: $\underset{\boldsymbol{Q}}{\boldsymbol{\sim}} \underset{\sim}{\boldsymbol{F}} \cdot \underline{\boldsymbol{\gamma}}$. This remark leads to the question of the contribution of the skins to the shear forces stiffness. Is it only the geometrical effect of the centroid or should $\boldsymbol{G}^{s}$ be part of the definition of $\underset{\sim}{\boldsymbol{F}}$ ?

Actually, sandwich panels with homogeneous core can be considered as a simpler case of laminated plates. These plates are usually made of highly anisotropic layers and a large literature is dedicated to their complex transverse shear behavior $[14,15,16,17]$. Consequently, some apply directly the simplest related theory (First Order Shear Deformation Theory) and assume uniform shear strain in both core and skins with a shear correction factor taken as 1. This leads to: $\underset{\sim}{\boldsymbol{F}}=t_{c} \boldsymbol{G}^{c}+2 t_{s} \boldsymbol{G}^{s}[18,19]$. Here, the stiffness of the skins affects the shear forces stiffness. Clearly this suggestion is not satisfying because when $\boldsymbol{G}^{c}$ becomes really small, one would expect the shear forces stiffness going to 0 since a sandwich panel without core is not a structure. Rigorously, applying FOSDT necessitates the derivation of shear correction factors. However the derivation of such factors seems still under question. For instance Birman and Bert [20] compared several approaches which give almost incompatible results especially when considering limit cases. Thus, if one wants to apply FOSDT, some clarifications are needed.

We conclude that, the contribution of the skins to the shear forces stiffness is controversial in the case of homogeneous cores.

\subsection{The case of cellular cores}

When dealing with cellular sandwich panels, the most common approach consists in finding a homogenized behavior for the core and using it in the formulas discussed in the previous section. Forgetting about the difficulties already pointed out, the question is now to find $\boldsymbol{G}^{c, \text { eff }}$ the effective transverse shear stiffness of the core. A first guess is to use classical results from 3D homogenization of a periodic medium ([21] among others). This may be possible for honeycomb cores but more complex geometries such as truss or folded cores are not periodic in Direction 3. Thus, alternative approaches are needed.

Based on the intuition that the skins put the core into transverse shear because of their relative inplane displacement Kelsey et al. [13] suggested to apply uniform displacement (resp. force) on the core replacing the action of the skins, in order to derive an upper (resp. lower) bound of the transverse shear stiffness of the core. This effective stiffness for the core, combined with Equation 1 leads to the shear forces stiffness. Although it is not fully justified (or lacking references), this method was broadly applied on honeycomb-like geometries ([22, 23, 24] among others). It was found rather efficient and even enabled the derivation of exact solutions in some configurations. However, for other core geometries, this approach yields loose bounds because it precludes any possible interaction between the skins and the core.

Two phenomena were identified and often mixed under the name of "skin effect". First, there is a kinematic incompatibility between the rigid skins and the strain in the core. Second, the skins can be distorted by the core.

The kinematic incompatibility was first considered by Penzien and Didriksson [25]. Later, Grediac [26] derived the bounds using finite elements releasing the assumption of piecewise uniform fields in the cellular core. It was also followed by several authors who gave a more detailed analysis of the incompatibility $[27,28,29]$. However, these works are specifically tailored for honeycomb geometries and assume fully rigid skins. Hence, they do not consider the out-of-plane skins distortion.

The skins distortion was pointed out in several works $[30,31,32,33]$ and is the main cause of a poor 
estimation of shear forces stiffness even when refining the bounds from Kelsey et al. [13]. In order to take into account this effect, two suggestions were made.

First, comparison with the full 3D model of the sandwich panel under cylindrical bending were performed $[31,34]$ and shear force stiffness was chosen to fit the plate model to the full simulation. The merit of such an approach is that it takes the problem as a whole and there is no surrogate for the core. However, it presents several limitations, the first being the high computational cost. There is also an arbitrary choice for the plate boundary problem to be solved (here a cylindrical bending).

Second, homogenization approaches were suggested. First, Shi and Tong [30] suggested to apply a uniform transverse shear strain on average in the core (as done in periodic homogenization schemes) to which are welded the skins. This enabled them to bring out the skins own flexural stiffness influence on the actual shear forces stiffness of honeycombs. Their approach was applied to several honeycomb geometries by Hohe [35] who calls it the direct homogenization method. Even if this approach is based on an acute intuition of the influence of the skins on the core, it remains limited to sandwich panel with thin skins and no theoretical justification was provided. Finally, Cecchi and Sab [36] suggested an extension to periodic plates of the method from Whitney [37] for deriving transverse shear distribution for laminated plates. This method is valid for any type of periodic plate (not only sandwich panels) and assumes that the plate is in cylindrical bending configuration when identifying shear forces stiffness. In a recent work from Lebée and Sab [1], it was demonstrated that this last assumption is too restrictive when dealing with heterogeneous plates such as laminated plates and that it is necessary to use a new general plate model: the Bending-Gradient model.

The Bending-Gradient theory was applied to a sandwich panel including a folded core in Lebée and Sab [33]. It turned out that the transverse shear behavior of the plate was mostly of Reissner-Mindlin type for a wide variety of configurations. The Bending-Gradient theory is a rather complex homogenization scheme to implement (see next section). Hence, starting from this theory, it is interesting to look for a simpler and direct homogenization scheme dedicated to sandwich panel which leads to the classical Reissner-Mindlin model.

\section{The Bending-Gradient model and its homogenization scheme}

Full details about the Bending-Gradient plate theory are provided in $[1,33,38]$. In this section we recall the main features of this plate theory and the related homogenization scheme.

\subsection{Summary of the Bending-Gradient plate model}

We consider a linear elastic plate which mid-plane is the $2 \mathrm{D}$ domain $\omega \subset \mathbb{R}^{2}$. Cartesian coordinates $\left(x_{1}, x_{2}, x_{3}\right)$ in the reference frame $\left(\underline{\hat{e}}_{1}, \underline{\hat{e}}_{2}, \underline{\hat{e}}_{3}\right)$ are used to describe macroscopic fields. At this stage, the microstructure of the plate is not specified.

The membrane stress $N_{\alpha \beta}$, the bending moment $M_{\alpha \beta}$, and shear forces $Q_{\alpha}$ are the usual generalized stresses for plates. Both $\underset{\sim}{N}$ and $\underset{\sim}{M}$ follow the classical symmetry of stress tensors: $N_{\alpha \beta}=N_{\beta \alpha}$. Moreover, we introduce an additional static unknown: the gradient of the bending moment $R_{\alpha \beta \gamma}=M_{\alpha \beta, \gamma}$. The 2D third-order tensor $\boldsymbol{R}$ complies with the following symmetry: $R_{\alpha \beta \gamma}=R_{\beta \alpha \gamma}$. It is possible to derive shear forces $\underline{\boldsymbol{Q}}$ from $\underline{\boldsymbol{R}}$ with: $Q_{\alpha}=R_{\alpha \beta \beta}$. This rewrites $\underline{\boldsymbol{Q}}=\underset{\sim}{\boldsymbol{i}} \therefore \underline{\boldsymbol{R}}$ where the triple contraction product $\therefore$ is defined as $i_{\alpha \beta \gamma \delta} R_{\delta \gamma \beta}$ and $\underset{\sim}{\boldsymbol{i}}$ is the identity for in-plane elasticity tensors $\left(i_{\alpha \beta \gamma \delta}=\frac{1}{2}\left(\delta_{\alpha \gamma} \delta_{\beta \delta}+\delta_{\alpha \delta} \delta_{\beta \gamma}\right)\right)$.

The main difference between Reissner-Mindlin and Bending-Gradient plate theories is that the BendingGradient plate theory enables the distinction between each component of the gradient of the bending moment whereas they are mixed into the shear forces with Reissner-Mindlin theory. In the case of highly 
anisotropic laminated plates this distinction is critical for deriving good estimate of the deflection and local transverse shear distribution through the thickness [38].

The full bending gradient $\underline{\boldsymbol{R}}$ has six components whereas $\underline{\boldsymbol{Q}}$ has two components. Thus, using the full bending gradient as static unknown introduces four additional static unknowns. More precisely: $R_{111}$ and $R_{222}$ are respectively the cylindrical bending part of shear forces $Q_{1}$ and $Q_{2}, R_{121}$ and $R_{122}$ are respectively the torsion part of these shear forces and $R_{112}$ and $R_{221}$ are linked to strictly self-equilibrated stresses. Equilibrium equations and stress boundary conditions are detailed in Appendix A.

Generalized stresses $\boldsymbol{N}, \boldsymbol{M}$, and $\boldsymbol{R}$ work respectively with the associated strain variables: $e_{\alpha \beta}$, the conventional membrane strain, $\chi_{\alpha \beta}$ the curvature and $\Gamma_{\alpha \beta \gamma}$ the third order tensor related to generalized shear strains. These strain fields must comply with the compatibility conditions and boundary conditions detailed in Appendix A.

Finally, assuming uncoupling between $(\underset{\sim}{\boldsymbol{N}}, \underset{\sim}{\boldsymbol{M}})$ and $\underline{\boldsymbol{R}}$ (see next section), the Bending-Gradient plate constitutive equations are written as:

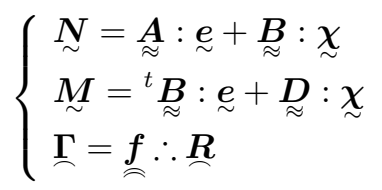

where $\underset{\approx}{\boldsymbol{A}}, \underset{\approx}{\boldsymbol{B}}, \underset{\approx}{\boldsymbol{D}})$ are conventional Kirchhoff-Love stiffness tensors. The transpose operation ${ }^{t} \bullet$ is applied to any order tensors as follows: $\left({ }^{t} A\right)_{\alpha \beta \ldots \psi \omega}=A_{\omega \psi \ldots \beta \alpha}$. The generalized shear compliance tensor $\underset{f}{\boldsymbol{f}}$ is a sixth order tensor ${ }^{2}$.

It was demonstrated that if $\underset{\sim}{\boldsymbol{f}}=\underset{\sim}{\boldsymbol{i}} \cdot \underset{\sim}{\boldsymbol{f}} \cdot \underset{\sim}{\boldsymbol{i}}=\left(i_{\alpha \beta \gamma \eta} f_{\eta \theta} i_{\theta \delta \epsilon \zeta}\right)$, then the Bending-Gradient model is strictly turned into a Reissner-Mindlin model. Then, Equation 2c reduces to $\underline{Q}={\underset{\sim}{\boldsymbol{f}}}^{-1} \cdot \underline{\boldsymbol{\gamma}}$. This is exactly the case for homogeneous plates where $\underset{\widetilde{f}}{\boldsymbol{f}} \underset{\sim}{\boldsymbol{i}} \cdot \frac{6 h}{5} \boldsymbol{G}^{-1} \underset{\sim}{\boldsymbol{i}}$ leads to $\underline{\boldsymbol{Q}}=5 / 6 h \boldsymbol{G} \cdot \underline{\boldsymbol{\gamma}}$. For this reason, the Bending-Gradient theory is seen as the extension to heterogeneous plates of the Reissner-Mindlin theory for homogeneous plates.

When the plate is periodic, the constitutive tensors $\underset{\sim}{\boldsymbol{A}} \underset{\sim}{\boldsymbol{B}} \underset{\sim}{\boldsymbol{D}}$ and $\underset{\approx}{\boldsymbol{f}}$ are derived using the homogenization scheme which will be discussed later on. However closed-form expressions exist when the plate is laminated (i.e.: the local constitutive equation is only function of $x_{3}$ ). Kirchhoff-Love stiffness are derived using the Classical Lamination Theory [39]:

$$
(\underset{\sim}{\boldsymbol{A}}, \underset{\approx}{\boldsymbol{B}}, \underset{\approx}{\boldsymbol{D}})=\int_{-\frac{h}{2}}^{\frac{h}{2}}\left(1, x_{3}, x_{3}^{2}\right) \underset{\approx}{\boldsymbol{c}}\left(x_{3}\right) d x_{3}
$$

where $\underset{\sim}{\boldsymbol{c}}\left(x_{3}\right)$ is the local plane-stress stiffness tensor $\underset{\sim}{\boldsymbol{c}}=\underset{\sim}{\boldsymbol{S}}{ }^{-1}$ is the inverse of the plane part of the 3D compliance $\underset{\approx}{\hat{\boldsymbol{S}}}={\underset{\approx}{\hat{\mathbb{C}}^{-1}}}^{-1}$. The generalized shear compliance tensor is then:

$$
\underset{\sim}{\boldsymbol{f}}=\int_{-\frac{h}{2}}^{\frac{h}{2}}\left(\int_{-\frac{h}{2}}^{x_{3}}\left({ }_{\sim}^{t} \underset{\sim}{\boldsymbol{b}}+z \underset{\sim}{\boldsymbol{d}}\right): \underset{\sim}{\boldsymbol{c}}(z) d z\right) \cdot{\underset{\sim}{\boldsymbol{G}}}^{-1}\left(x_{3}\right) \cdot\left(\int_{-\frac{h}{2}}^{x_{3}} \underset{\sim}{\boldsymbol{c}}(z):(\underset{\sim}{\boldsymbol{b}}+z \underset{\sim}{\boldsymbol{d}}) d z\right) d x_{3}
$$

where $(\underset{\sim}{\boldsymbol{a}}, \underset{\sim}{\boldsymbol{b}}, \underset{\sim}{\boldsymbol{d}})$ are the Kirchhoff-Love compliances tensors expressing $(\underset{\sim}{e}, \underset{\sim}{\boldsymbol{\chi}})$ in terms of $(\underset{\sim}{\boldsymbol{N}}, \boldsymbol{\sim})$ by inverting $2 \mathrm{a}$ and $2 \mathrm{~b}$ and $\boldsymbol{G}$ is the local transverse shear stiffness [1].

2. $f_{\alpha \beta \gamma \delta \epsilon \zeta}$ follows major symmetry: $f_{\alpha \beta \gamma \delta \epsilon \zeta}=f_{\zeta \epsilon \delta \gamma \beta \alpha}$ and minor symmetry $f_{\alpha \beta \gamma \delta \epsilon \zeta}=f_{\beta \alpha \gamma \delta \epsilon \zeta}$. Thus there are only 21 independent components 


\subsection{Homogenization scheme}

In this section, the derivation of $\underset{\approx}{\boldsymbol{A}}, \underset{\approx}{\boldsymbol{B}}, \underset{\approx}{\boldsymbol{D}}$ and $\boldsymbol{f}$ is detailed in the general case of a periodically heterogeneous plate.

Let us consider a plate generated by periodicity of a unit-cell $Y$ (Figure 2). The upper face $\partial Y_{3}^{+}$and the lower face $\partial Y_{3}^{-}$are traction free and the lateral faces $\partial Y_{l}$ must fulfill periodicity conditions. $A_{Y}$ is the area of the unit-cell cross section with the plate mid-plane. In the following, the plate is assumed invariant by $\pi$-angle rotation with respect to a vertical axis so that uncoupling between $\boldsymbol{N}, \boldsymbol{M}$ and $\boldsymbol{R}$ is ensured [33]. This invariance is the generalization to periodic plates of the monoclinic symmetry classically made with laminated plates and invoked in Section 2 for the skins and a homogeneous core.

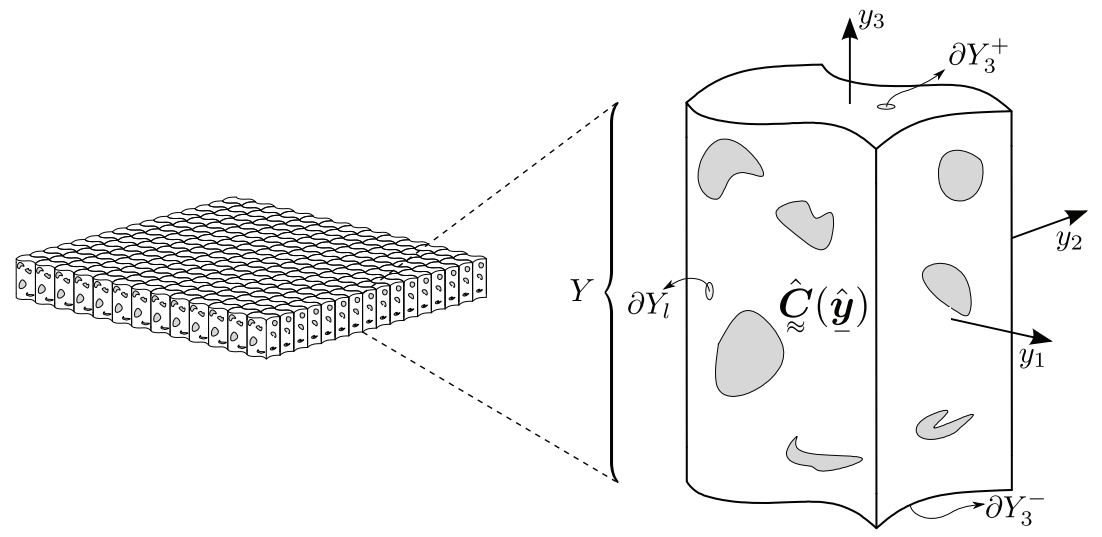

Figure 2. The plate unit-cell

In order to derive a homogenized behavior of the heterogeneous plate, it is assumed that macroscopic fields $(\underset{\sim}{N}, \underset{\sim}{\boldsymbol{M}}, \boldsymbol{R})$ and their associated stress energy density vary slowly with respect to the size of the unit-cell. Then, using Hill-Mandel principle, the average of the local energy in the unit-cell (microscopic energy) is assumed equal to the plate energy (macroscopic energy). This is equivalent to setting:

$$
\frac{1}{2}\left(\underset{\sim}{\boldsymbol{N}}: \underset{\sim}{\boldsymbol{a}}: \underset{\sim}{\boldsymbol{N}}+2 \underset{\sim}{\boldsymbol{N}}: \underset{\sim}{\boldsymbol{b}}: \underset{\sim}{\boldsymbol{M}}+\underset{\sim}{\boldsymbol{M}}: \underset{\sim}{\boldsymbol{d}}: \underset{\sim}{\boldsymbol{M}}+{ }^{t} \underline{\boldsymbol{R}} \therefore \underset{\sim}{\boldsymbol{f}} \therefore: \underline{\boldsymbol{R}}\right)=\frac{1}{2}\left\langle\hat{\boldsymbol{\sigma}}^{B G}(\underline{\hat{\boldsymbol{y}}}): \underline{\sim}_{\boldsymbol{S}}^{\hat{\boldsymbol{y}}}\left(\underline{\hat{\boldsymbol{\sigma}}^{B G}}(\underline{\hat{\boldsymbol{y}}})\right\rangle\right.
$$

where

$$
\langle f\rangle=\frac{1}{A_{Y}} \int_{Y} f d \underline{\hat{\boldsymbol{y}}}
$$

is the normalized average (surface average) on the unit-cell and ${\underset{\sim}{\hat{\sigma}}}^{B G}$ is an approximation of the local stress field in the unit-cell generated by the macroscopic static unknowns $(\boldsymbol{N}, \underline{\boldsymbol{N}}, \underline{\boldsymbol{R}})$. More precisely, the field localization $\hat{\boldsymbol{\sigma}}^{B G}$ is a linear application from $(\underset{\sim}{\boldsymbol{N}}, \underset{\sim}{\boldsymbol{M}}, \underset{\boldsymbol{R}}{\boldsymbol{R}})$ to a $3 \mathrm{D}$ stress field in the unit-cell which is detailed as follows:

$$
\stackrel{\hat{\boldsymbol{\sigma}}}{ }^{B G}=\underline{\sim}^{(N)}(\underline{\hat{\boldsymbol{y}}})+\underline{\sim}^{(M)}(\underline{\hat{\boldsymbol{y}}})+\stackrel{\sim}{\sim}^{(R)}(\underline{\hat{\boldsymbol{y}}})
$$

where $\hat{\sim}^{(N)}, \hat{\boldsymbol{\sigma}}^{(M)}$, and ${\underset{\sim}{(R)}}^{(R)}$ are 3D stress fields generated respectively by $\underset{\sim}{\boldsymbol{N}}, \boldsymbol{M}$ and $\underline{\boldsymbol{R}}$ : 


$$
\left\{\begin{array}{l}
\sigma_{i j}^{(N)}=s_{i j \alpha \beta}^{(N)}(\underline{\hat{\boldsymbol{y}}}) N_{\beta \alpha} \\
\sigma_{i j}^{(M)}=s_{i j \alpha \beta}^{(M)}(\underline{\hat{\boldsymbol{y}}}) M_{\beta \alpha} \\
\sigma_{i j}^{(R)}=s_{i j \alpha \beta \gamma}^{(R)}(\underline{\hat{\boldsymbol{y}}}) R_{\gamma \beta \alpha}
\end{array}\right.
$$

and $s_{i j \alpha \beta}^{(N)}(\underline{\hat{\boldsymbol{y}}}), s_{i j \alpha \beta}^{(M)}(\underline{\hat{\boldsymbol{y}}})$ and $s_{i j \alpha \beta \gamma}^{(R)}(\underline{\hat{\boldsymbol{y}}})$ are localization field tensors (unit load fields).

The determination of localization fields necessitates the resolution of auxiliary unit-cell problems. First, the Kirchhoff-Love homogenization auxiliary problem was suggested by Caillerie [40] and enables the derivation of the effective Kirchhoff-Love membrane and flexural moduli of the periodic plate $\underset{\approx}{\boldsymbol{A}} \underset{\approx}{\boldsymbol{B}}$ and $\underset{\approx}{\boldsymbol{D}}$, as well as the local 3D stresses ${\underset{\sim}{\hat{\boldsymbol{s}}}}^{(e)}(\hat{\boldsymbol{y}})$ and $\underset{\sim}{\hat{\boldsymbol{s}}^{(\chi)}}(\hat{\boldsymbol{y}})$ related to membrane strains and curvature. Then a generalized shear auxiliary problem is defined, using Kirchhoff-Love localization fields as loading inputs, in order to derive the generalized shear compliance $\boldsymbol{f}$ and $\underline{\hat{s}}^{(R)}(\underline{\hat{y}})$.

\subsubsection{Kirchhoff-Love auxiliary problem}

Kirchhoff-Love homogenization of periodic plates was first proposed by Caillerie [40] and followed by Kohn and Vogelius [41]. The auxiliary problem is stated as follows:

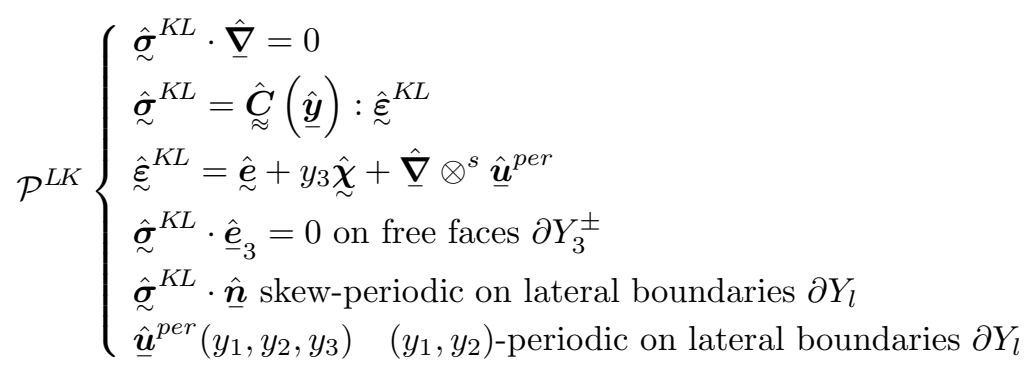

Basically it enforces the membrane strains $\underset{\sim}{e}$ and the curvatures $\underset{\sim}{\chi}$ on average on the unit-cell while taking into account periodicity in the $\left(x_{1}, x_{2}\right)$-plane and traction-free conditions on the upper and lower faces of the plate. In Equation 6, $\underset{\sim}{\hat{e}}$ and $\underset{\sim}{\hat{\chi}}$ denote the extension to 3D of the 2D tensors:

$$
\hat{e}=\left(\begin{array}{ccc}
e_{11} & e_{12} & 0 \\
e_{21} & e_{22} & 0 \\
0 & 0 & 0
\end{array}\right) \quad \text { and } \quad \hat{\sim}=\left(\begin{array}{ccc}
\chi_{11} & \chi_{12} & 0 \\
\chi_{21} & \chi_{22} & 0 \\
0 & 0 & 0
\end{array}\right)
$$

Furthermore, there is a Hill-lemma relation for plate [40,42] which gives the following definition for $\boldsymbol{N}$ and $\underset{\sim}{M}$ as the average of stress fields in the unit-cell:

$$
N_{\alpha \beta}=\left\langle\sigma_{\alpha \beta}^{K L}\right\rangle \text { and } M_{\alpha \beta}=\left\langle y_{3} \sigma_{\alpha \beta}^{K L}\right\rangle
$$

Solving the problem for each individual component of $\underset{\sim}{e}$ and $\underset{\sim}{\chi}$ leads to the localization stress fields $\underset{\sim}{\hat{\sim}^{(e)}}$ and $\underset{\sim}{\stackrel{s}{(}(\chi)}$. Kirchhoff-Love plate moduli are then evaluated as follows:

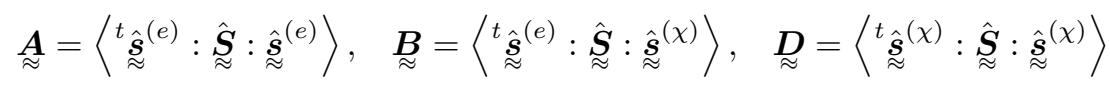

In addition one can derive the following variational principle related to problem $\mathcal{P}^{L K}$ :

- Static approach: The set of statically compatible fields $S C^{L K}$ is the set of stress fields $\hat{\sim}$ which fulfill equilibrium equation (6a), boundary conditions (6d) and periodicity conditions (6e). 
The stress solution $\hat{\sim}^{L K}$ is the one that minimizes the complementary energy per unit surface $P^{* L K}$ :

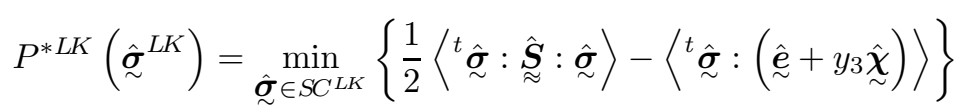

- Kinematic approach: The set of kinematically compatible fields $K C^{L K}$ is the set of strain fields $\underset{\sim}{\hat{\varepsilon}}$ which fulfill compatibility conditions (6c) and periodicity conditions (6f).

The strain solution $\underset{\sim}{\hat{\varepsilon}^{L K}}$ is the one that minimizes the potential energy per unit surface $P^{L K}$ :

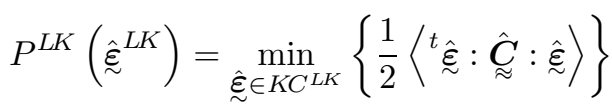

- Clapeyron's formula: The following relation holds for the solution:

$$
P^{L K}\left({\underset{\sim}{\tilde{\varepsilon}}}^{L K}\right)=-P^{* L K}\left({\underset{\sim}{\hat{\sigma}}}^{L K}\right)=\frac{1}{2}(\underset{\sim}{e}: \underset{\sim}{\boldsymbol{A}}: \underset{\sim}{\boldsymbol{e}}+2 \underset{\sim}{e}: \underset{\sim}{\boldsymbol{B}}: \underset{\sim}{\chi}+\underset{\sim}{\underset{\sim}{\boldsymbol{D}}: \underset{\sim}{\boldsymbol{\sim}})})
$$

\subsubsection{The generalized shear auxiliary problem}

The generalized shear auxiliary problem on the unit-cell is defined as:

$$
\mathcal{P}^{B G}\left\{\begin{array}{l}
\hat{\boldsymbol{\sigma}}^{(R)} \cdot \underline{\hat{\boldsymbol{v}}}+\underline{\hat{\boldsymbol{f}}}^{(R)}(\underline{\hat{\boldsymbol{y}}})=0 \\
\hat{\boldsymbol{\sigma}}^{(R)}=\hat{\boldsymbol{C}}(\underline{\hat{\boldsymbol{y}}}):\left(\hat{\boldsymbol{\boldsymbol { v }}} \otimes^{s} \underline{\hat{\boldsymbol{u}}}^{(R)}\right) \\
\hat{\boldsymbol{\sigma}}^{(R)} \cdot \underline{\hat{\boldsymbol{\theta}}}_{3}=0 \text { on free faces } \partial Y_{3}^{ \pm} \\
\hat{\boldsymbol{\boldsymbol { \sigma }}}^{(R)} \cdot \underline{\hat{\boldsymbol{n}}} \text { skew-periodic on lateral boundaries } \partial Y_{l} \\
\underline{\hat{\boldsymbol{u}}}^{(R)}\left(y_{1}, y_{2}, y_{3}\right) \quad\left(y_{1}, y_{2}\right) \text {-periodic on lateral boundaries } \partial Y_{l}
\end{array}\right.
$$

where

$$
f_{i}^{(R)}=\left(s_{i \gamma \delta \varepsilon}^{(e)} b_{\varepsilon \delta \beta \alpha}+s_{i \gamma \delta \varepsilon}^{(\chi)} d_{\varepsilon \delta \beta \alpha}\right) R_{\alpha \beta \gamma}
$$

is the body force generated by linear variations of $\boldsymbol{M}$, namely its gradient: $\boldsymbol{R}$. More precisely, it is derived taking the 3D divergence of the local stress $\hat{\boldsymbol{\sigma}}^{(M)}$, where the bending moment is $\underset{\sim}{\boldsymbol{M}}=\underline{\boldsymbol{R}} \cdot \boldsymbol{y}$. Since $\hat{\boldsymbol{f}}^{(R)}$ involves localization fields previously derived in Kirchhoff-Love auxiliary problem, one must perform this computation first. Solving $\mathcal{P}^{B G}$ for each component of $\underline{\boldsymbol{R}}$ leads to the localization stress field $s_{i j \alpha \beta \gamma}^{(R)}$ related to $\underline{\boldsymbol{R}}$. It is then possible to identify the Bending-Gradient compliance tensor as:

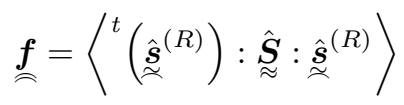

Whereas it was possible to define $\underset{\sim}{N}$ and $\underset{\sim}{M}$ as resultant of local stress fields, there is no such a direct relation for the gradient of the bending moment $\underline{\boldsymbol{R}}$. However, it is possible to demonstrate:

$$
\left\langle\sigma_{\alpha 3}^{(R)}\right\rangle=i_{\alpha \beta \gamma \delta} R_{\delta \gamma \beta}=Q_{\alpha} \quad \text { and } \quad\left\langle\sigma_{33}^{(R)}\right\rangle=0
$$

In addition one can derive the following variational principle related to problem $\mathcal{P}^{B G}$ :

- Static approach: The set of statically compatible fields $S C^{B G}$ is the set of stress fields $\hat{\sim}$ which fulfill equilibrium equation (9a), boundary conditions (9c) and periodicity conditions (9d).

The stress solution $\hat{\boldsymbol{\sigma}}^{(R)}$ is the one that minimizes the complementary energy $P^{* B G}$ : 


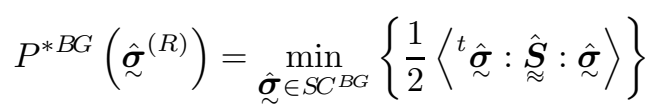

- Kinematic approach: The set of kinematically compatible fields $K C^{B G}$ is the set of strain fields $\underset{\sim}{\hat{\varepsilon}}$ which derive from a displacement field which fulfills periodicity conditions (9e).

The strain solution ${\underset{\sim}{(R)}}^{(R)}$ is the one that minimizes the potential energy $P^{B G}$ :

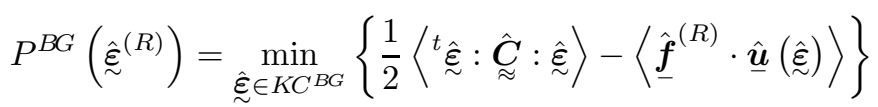

- Clapeyron's formula: The following relation holds for the solution:

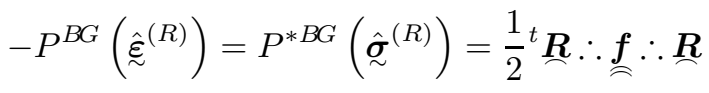

\section{The in-plane behavior of sandwich panels}

The Kirchhoff-Love behavior of sandwich panels is well-known, especially with homogeneous cores. In this section, we recall these results and derive bounds when the sandwich panel is cellular.

\subsection{The case of homogeneous cores}

Let us consider first a sandwich panel with a homogeneous core. Direct application of the Classical Lamination Theory (Equation 3) leads to the following Kirchhoff-Love stiffness tensors:

$$
\underset{\approx}{\boldsymbol{A}}=2 t_{s} \underset{\approx}{\boldsymbol{c}^{s}}+t_{c} \underset{\approx}{\boldsymbol{c}^{c}}, \quad \underset{\approx}{\boldsymbol{B}}=\underset{\approx}{\mathbf{0}}, \quad \underset{\approx}{\boldsymbol{D}}=\frac{h^{3}-t_{c}^{3}}{12} \underset{\approx}{\boldsymbol{c}^{s}}+\frac{t_{c}^{3}}{12} \boldsymbol{c}^{c}
$$

Then the contrast assumption consists in neglecting the contribution of the core to these stiffness tensors. In the present case, it is equivalent to assuming:

$$
\forall \underset{\sim}{e}, \quad \underset{\sim}{e}: t_{c}{\underset{\sim}{c}}^{c}: \underset{\sim}{e} \ll \underset{\sim}{e}: 2 t_{s} \underset{\sim}{\boldsymbol{c}^{s}}: \underset{\sim}{\boldsymbol{e}}
$$

where $e_{\alpha \beta}$ is any symmetric second order tensor in $2 \mathrm{D}$. The stiffness tensors become then:

$$
\underset{\approx}{\boldsymbol{A}}=2 t_{s} \underset{\approx}{\boldsymbol{c}^{s}}, \quad \underset{\approx}{\boldsymbol{B}}=\underset{\approx}{\mathbf{0}}, \quad \underset{\approx}{\boldsymbol{D}}=\frac{h^{3}-t_{c}^{3}}{12} \boldsymbol{\sim}^{s}
$$

Additionally one can assume also thin skins. The flexural stiffness is simplified into the well known formula:

$$
\underset{\approx}{\boldsymbol{D}}=\frac{t_{s}}{2}\left(t_{c}+t_{s}\right)^{2} \underset{\approx}{\boldsymbol{c}^{s}}
$$

These very basic results will be extended to the case of cellular sandwich panel in the following.

\subsection{The case of cellular cores}

The homogenization scheme provided in Section 3.2.1 is designed for any periodic plate. Now we specify its application to a cellular sandwich panel. The unit-cell $Y$ was detailed in Figure 1. It is separated in three parts, the upper skin $Y^{s+}$, the lower skin $Y^{s-}$ and the core $Y^{c}\left(Y=Y^{s+} \cup Y^{c} \cup Y^{s-}\right)$. We apply variational principles for the Kirchhoff-Love auxiliary problem to this unit-cell in order to derive bounds. Finally, this enables us to formulate the contrast assumption for cellular sandwich panels. 


\subsubsection{Bounds for Kirchhoff-Love stiffnesses of cellular sandwich panels}

The derivation of bounds is done in two steps. First, compatible trial fields are defined in each part of the unit-cell. Then, the related energies are derived and compared to the exact solution.

4.2.1.1. Lower bound The derivation of the lower bound is based on the conventional assumption that only the skins are put under traction and compression when bending the sandwich panel. We choose a statically compatible stress $\boldsymbol{\sigma}^{-} \in S C^{L K}$ which is null in the core and plane-stress in the skins:

$$
\boldsymbol{\sigma}^{-}=\left\{\begin{array}{ll}
\underset{\sim}{\mathbb{s}^{s}}:\left(\underset{\sim}{\boldsymbol{e}}+x_{3} \underset{\sim}{\boldsymbol{\chi}}\right) & \text { on } Y^{s \pm} \\
\underset{\sim}{\mathbf{0}} & \text { on } Y^{c}
\end{array} \quad \text { and } \quad \sigma_{i 3}^{-}=0 \quad \text { on } Y\right.
$$

The complementary energy (7) related to $\hat{\sim}^{-}$involves only the skins and is written as:

$$
P^{* L K}\left({\underset{\sim}{\boldsymbol{\sigma}}}^{-}\right)=-\frac{1}{2}\left\langle{ }^{t}\left(\underset{\sim}{e}+y_{3} \underset{\sim}{\chi}\right):{\underset{\sim}{c}}^{s}:\left(\underset{\sim}{e}+y_{3} \underset{\sim}{\chi}\right)\right\rangle_{s}
$$

where $\langle f\rangle_{s}$ is the normalized average restricted to the skins:

$$
\langle f\rangle_{s}=\frac{1}{A_{Y}} \int_{Y^{s+} \bigcup Y^{s-}} f d \underline{\hat{\boldsymbol{y}}}
$$

Application of the static approach of variational principles leads to:

$$
w^{L K, s}<\frac{1}{2}(\underset{\sim}{e}: \underset{\sim}{\boldsymbol{A}}: \underset{\sim}{e}+2 \underset{\sim}{e}: \underset{\sim}{\boldsymbol{B}}: \underset{\sim}{\chi}+\underset{\sim}{\underset{\sim}{\boldsymbol{D}}: \underset{\sim}{\boldsymbol{D}}: \underset{\sim}{\chi})}
$$

where we defined the contribution of the skins to the energy as:

$$
w^{L K, s}=\frac{1}{2}\left\langle{ }^{t}\left(\underset{\sim}{e}+y_{3} \underset{\sim}{\chi}\right):{\underset{\sim}{c}}^{s}:\left(\underset{\sim}{e}+y_{3} \underset{\sim}{\chi}\right)\right\rangle_{s}
$$

from which the lower bound for Kirchhoff-Love stiffness tensors is extracted:

$$
\underset{\approx}{\underset{\sim}{A}}=2 t_{s}{\underset{\approx}{s}}^{s}, \quad \underset{\approx}{\boldsymbol{B}^{-}}=\underset{\approx}{\mathbf{0}} \quad \underset{\approx}{\boldsymbol{D}^{-}}=\frac{h^{3}-t_{c}^{3}}{12}{\underset{\approx}{c}}^{s}
$$

4.2.1.2. Upper bound For the upper bound, we choose the kinematically compatible strain field $\hat{\boldsymbol{\varepsilon}}^{+} \in$ $K C^{L K}$ which is plane-strain in the core and plane-stress in the skins:

$$
\hat{\sim}^{+}=\left\{\begin{array}{l}
\underset{\sim}{\hat{e}}+x_{3} \underset{\sim}{\hat{\boldsymbol{\chi}}}+\left(\underset{\sim}{\boldsymbol{P}}:\left(\underset{\sim}{\boldsymbol{e}}+x_{3} \underset{\sim}{\boldsymbol{\chi}}\right)\right) \underline{\hat{e}}_{3} \otimes \underline{\underline{\boldsymbol{e}}}_{3} \quad \text { on } Y^{s \pm} \\
\underset{\sim}{\hat{e}}+x_{3} \underset{\sim}{\hat{\boldsymbol{\chi}}} \text { on } Y^{c}
\end{array}\right.
$$

where $P_{\alpha \beta}^{s}=-\frac{C_{33 \alpha \beta}^{s}}{C_{3333}^{s}}$ is the correction which takes into account the out-of-plane Poisson's effect in the skins. Assuming plane-stress in the core would lead to incompatibility of strain fields at the skin/core interface in the general case. The potential energy (8) related to $\underset{\sim}{\hat{\varepsilon}^{+}}$is written as:

$$
P^{L K}\left({\underset{\sim}{\hat{\varepsilon}}}^{+}\right)=w^{L K, s}+\frac{1}{2}\left\langle{ }^{t}\left(\underset{\sim}{\hat{e}}+y_{3} \underset{\sim}{\hat{\chi}}\right):{\underset{\sim}{\hat{C}^{c}}}^{c}:\left(\underset{\sim}{\hat{e}}+y_{3} \underset{\sim}{\hat{\chi}}\right)\right\rangle_{c}
$$

where $\langle f\rangle_{c}$ is the normalized average restricted to the core:

$$
\langle f\rangle_{c}=\frac{1}{A_{Y}} \int_{Y^{c}} f d \underline{\hat{y}}
$$


Only the plane part of $\underset{\approx}{C}$ is involved in the second term of $P^{L K}$ since $e_{i 3}=\chi_{i 3}=0$ :

$$
P^{L K}\left(\underset{\hat{\boldsymbol{\varepsilon}}^{+}}{\hat{\sim}^{+}}\right)=w^{L K, s}+\frac{1}{2}\left\langle{ }^{t}\left(\underset{\sim}{\boldsymbol{e}}+y_{3} \underset{\sim}{\boldsymbol{\chi}}\right):{\underset{\approx}{c}}^{c}:\left(\underset{\sim}{\boldsymbol{e}}+y_{3} \underset{\sim}{\boldsymbol{\chi}}\right)\right\rangle_{c}
$$

Application of the kinematic approach of variational principles leads to:

$$
\frac{1}{2}(\underset{\sim}{\boldsymbol{e}}: \underset{\approx}{\boldsymbol{A}}: \underset{\sim}{\boldsymbol{e}}+2 \underset{\sim}{\boldsymbol{e}}: \underset{\approx}{\boldsymbol{B}}: \underset{\sim}{\boldsymbol{\chi}}+\underset{\sim}{\boldsymbol{\chi}}: \underset{\approx}{\boldsymbol{D}}: \underset{\sim}{\boldsymbol{\chi}})<w^{L K, s}+w^{L K, c}
$$

where we defined the contribution of the core to the energy as:

$$
w^{L K, c}=\frac{1}{2}\left\langle{ }^{t}\left(\underset{\sim}{e}+y_{3} \underset{\sim}{\boldsymbol{\chi}}\right):{\underset{\approx}{c}}^{c}:\left(\underset{\sim}{e}+y_{3} \underset{\sim}{\chi}\right)\right\rangle_{c}
$$

We have the following Kirchhoff-Love stiffness tensors for the upper bound:

$$
\underset{\approx}{\boldsymbol{A}^{+}}=2 t_{s} \underset{\approx}{\boldsymbol{c}^{s}}+\langle\underset{\approx}{\boldsymbol{C}}\rangle_{c}, \quad \underset{\approx}{\boldsymbol{B}^{+}}=\left\langle y_{3} \underset{\approx}{\boldsymbol{C}^{c}}\right\rangle_{c} \text { and } \quad \underset{\approx}{\boldsymbol{D}^{+}}=\frac{h^{3}-t_{c}^{3}}{12}{\underset{\approx}{\boldsymbol{c}}}^{s}+\left\langle y_{3}^{2} \underset{\approx}{\boldsymbol{C}^{c}}\right\rangle_{c}
$$

Here, the core contribution is the upper bound for periodic plates from Kolpakov and Sheremet [43] applied directly to the core alone.

\subsubsection{Definition of the contrast assumption}

The derivation of bounds led to the following range for the energy:

$$
w^{L K, s}<\frac{1}{2}(\underset{\sim}{\boldsymbol{e}}: \underset{\approx}{\boldsymbol{A}}: \underset{\sim}{\boldsymbol{e}}+2 \underset{\sim}{\boldsymbol{e}}: \underset{\approx}{\boldsymbol{B}}: \underset{\sim}{\boldsymbol{\chi}}+\underset{\sim}{\boldsymbol{\chi}}: \underset{\approx}{\boldsymbol{D}}: \underset{\sim}{\boldsymbol{\chi}})<w^{L K, s}+w^{L K, c}
$$

The contrast assumption consists in neglecting the contribution of the core:

which is equivalent to:

$$
\forall(\underset{\sim}{\boldsymbol{e}}, \underset{\sim}{\boldsymbol{\chi}}), w^{L K, c}(\underset{\sim}{\boldsymbol{e}}, \underset{\sim}{\boldsymbol{\chi}}) \ll w^{L K, s}(\underset{\sim}{\boldsymbol{e}}, \underset{\sim}{\boldsymbol{\chi}})
$$

$$
\forall \underset{\sim}{e}, \quad \underset{\sim}{e}:\langle\underset{\approx}{C}\rangle_{c}: \underset{\sim}{e} \ll 2 t_{s} \underset{\sim}{e}: \underset{\sim}{c^{s}}: \underset{\sim}{e}
$$

This result is slightly different from the case of homogeneous core because it was not possible to assume plane-stress in the core. This is further discussed in Section 6.

Finally, under the contrast assumption the actual Kirchhoff-Love stiffness of the sandwich panel is equated to the lower bound and the related local stress field is considered as a good approximation at first order of the actual stress distribution.

\section{The transverse shear behavior of sandwich panels}

Knowing the in-plane behavior of the sandwich panel enables us now to derive its transverse shear behavior.

\subsection{The case of homogeneous cores}

In general, for 3-layer plates the Bending-Gradient model does not reduce to the Reissner-Mindlin model. However, it is possible to demonstrate that it is the case under the contrast assumption.

Direct application of Equation 4 and assumption (12) for a sandwich panel with homogeneous core leads to: 


$$
\underset{\approx}{\boldsymbol{f}}=\frac{k_{s}^{2}}{t_{c}^{2}} \underset{\sim}{\boldsymbol{i}} \cdot\left(t_{c} \underset{\sim}{\boldsymbol{g}}{ }^{c}+l_{s} t_{s}{\underset{\sim}{\boldsymbol{g}}}^{s}\right) \cdot \underset{\approx}{\boldsymbol{i}}, \text { where } k_{s}=\frac{3}{2} \frac{t_{c}\left(h^{2}-t_{c}^{2}\right)}{h^{3}-t_{c}^{3}} \text { and } l_{s}=\frac{10 t_{c}^{2}+25 t_{c} t_{s}+16 t_{s}^{2}}{15\left(t_{c}+t_{s}\right)^{2}}
$$

where ${\underset{\sim}{\boldsymbol{g}}}^{c}=\left(\boldsymbol{G}^{c}\right)^{-1}$ and ${\underset{\sim}{\boldsymbol{g}}}^{s}=\left(\boldsymbol{G}^{s}\right)^{-1}$ are the transverse shear compliances. The factorization of $\underset{\widetilde{f}}{\boldsymbol{f}}$ indicates that the constitutive equation is turned into a Reissner-Mindlin model. The corresponding shear forces stiffness writes as:

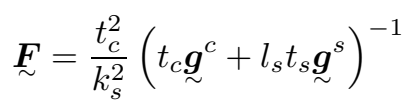

There is no a priori reason for neglecting the skins contribution since the contrast hypothesis involves only the plane part of 3D stiffnesses (Equation 12). For instance with carbon fiber reinforced composite skins, the transverse shear stiffness of the skins is quite low (about $1 / 25$ of the longitudinal stiffness).

With this result, when the thickness of the core goes to 0 we have $\underset{\sim}{\boldsymbol{F}}=\frac{5 \boldsymbol{h}}{6} \boldsymbol{G}^{s}$ and when $\boldsymbol{g}^{c}$ goes to the infinity, the shear forces stiffness goes to zero which is much more satisfying than FOSDT and classical approaches. Finally, if the skins are thin and their constitutive material is isotropic, their contribution is negligible. In this case, Equation 15 simplifies into:

$$
\underset{\sim}{\boldsymbol{F}}=\frac{t_{c}}{k_{s}^{2}} \boldsymbol{G}^{c}
$$

and we get the original result from Kelsey et al. [13].

\subsection{A direct homogenization scheme for cellular sandwich panel shear forces stiffness}

When dealing with cellular sandwich panels, stress distributions related to the in-plane behavior were derived in Section 4.2.1.1. Once the Kirchhoff-Love stress is defined, it is possible to apply the second step of the Bending-Gradient homogenization scheme (Problem 9).

Under the contrast assumption, the stress derived for the lower bound (Equation 13) makes a good approximation for the actual stress in the sandwich panel. The body load definition (Equation 10) applied to this stress distribution becomes:

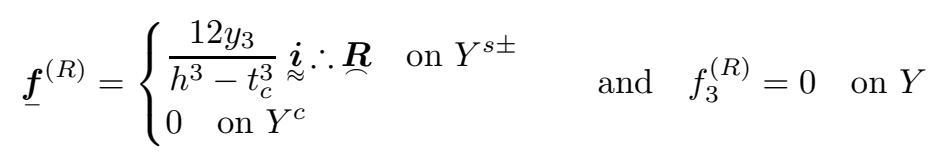

where we identify directly shear forces: $\underline{Q}=\underset{\sim}{\boldsymbol{i}} \therefore \underline{\boldsymbol{R}}$. Thus, with cellular sandwich panels under contrast assumption the Bending-Gradient model is also turned into a Reissner-Mindlin model.

Accordingly, we define the body force related to shear forces as:

$$
\underline{\boldsymbol{f}}^{(Q)}=\left\{\begin{array}{ll}
\frac{12 y_{3}}{h^{3}-t_{c}^{3}} \underline{\boldsymbol{Q}} & \text { on } Y^{s \pm} \\
0 \text { on } Y^{c}
\end{array} \quad \text { and } f_{3}^{(Q)}=0 \text { on } Y\right.
$$

Basically, it enforces volume forces in the skins so that the core is put into shearing exactly as suggested in sandwich theory (Figure 3).

Exactly as for $\underset{\boldsymbol{R}}{\boldsymbol{R}}$, the 3D stress field ${\underset{\sim}{\boldsymbol{\sigma}}}^{(Q)}$ related to shear forces is the stress field which equilibrates $\hat{\hat{\boldsymbol{f}}}^{(Q)}$ and the shear force auxiliary problem on the unit-cell is defined as: 


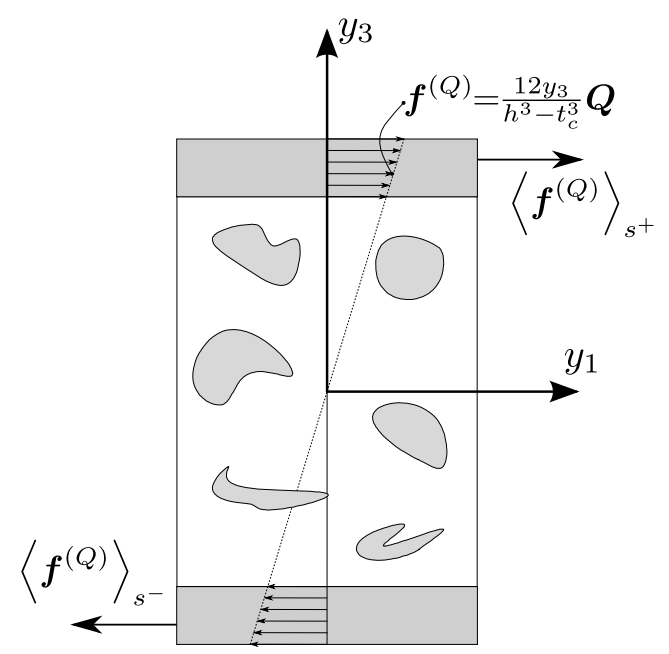

Figure 3. Shear force loading $\underline{\boldsymbol{f}}^{(Q)}$ for a sandwich panel under contrast assumption

$$
\mathcal{P}^{R M}\left\{\begin{array}{l}
\hat{\boldsymbol{\sigma}}^{(Q)} \cdot \hat{\hat{\boldsymbol{\nabla}}}+\hat{\hat{\boldsymbol{f}}}^{(Q)}(\underline{\hat{\boldsymbol{y}}})=0 \\
\hat{\boldsymbol{\sigma}}^{(Q)}=\hat{\boldsymbol{C}}(\hat{\boldsymbol{y}}):\left(\underline{\hat{\boldsymbol{v}}} \otimes^{s} \underline{\hat{\boldsymbol{u}}}^{(Q)}\right) \\
\hat{\boldsymbol{\boldsymbol { \sigma }}}^{(Q)} \cdot \underline{\hat{\boldsymbol{e}}}_{3}=0 \text { on free faces } \partial Y_{3}^{ \pm} \\
\hat{\boldsymbol{\sigma}}^{(Q)} \cdot \underline{\hat{\boldsymbol{n}}} \text { skew-periodic on lateral boundaries } \partial Y_{l} \\
\underline{\hat{\boldsymbol{u}}}^{(Q)}\left(y_{1}, y_{2}, y_{3}\right) \quad\left(y_{1}, y_{2}\right) \text {-periodic on lateral boundaries } \partial Y_{l}
\end{array}\right.
$$

This problem is Cecchi and Sab [36] auxiliary problem for deriving shear forces stiffness applied to a sandwich panel under contrast assumption without assuming a priori cylindrical bending. Again, solving $\mathcal{P}^{R M}$ leads to the localization stress field $s_{i j \alpha}^{(Q)}(\underline{\hat{y}})$ associated to shear forces, obtained by linear combination:

$$
\underline{\sim}^{(Q)}=\underline{\underline{\hat{s}}}^{(Q)}(\underline{\hat{\boldsymbol{y}}}) \cdot \underline{\boldsymbol{Q}}
$$

It is then possible to identify the shear forces compliance tensor with:

$$
\underset{\sim}{\boldsymbol{f}}=\left\langle{\underset{\sim}{\hat{\boldsymbol{s}}}}^{(Q)}: \underset{\sim}{\hat{\boldsymbol{S}}}: \stackrel{\underline{\underline{\boldsymbol{s}}}}{(Q)}^{(Q)}\right\rangle
$$

The variational principles related to $\mathcal{P}^{R M}$ are strictly identical to those introduced in Section 3.2.2 replacing " $R$ " by " $Q$ ".

Finally, the contrast assumption enables a direct derivation of shear forces stiffness with $\hat{\boldsymbol{f}}^{(Q)}$ loading and do not request the derivation of Kirchhoff-Love localization fields. Thus $\mathcal{P}^{R M}$ defines a direct homogenization scheme for sandwich panels under contrast assumption.

\subsection{Bounds for sandwich panels shear forces stiffness}

Now it is possible to provide justification for the bounds commonly used in sandwich theory. Since the actual shear forces loading is known for a sandwich panel, we apply variational principles to the shear forces auxiliary problem $\mathcal{P}^{R M}$. 


\subsubsection{Voigt and Reuss bounds}

Assuming rigid displacement in the skins and uniform shear strain in the core enables the derivation of a Voigt type upper bound for sandwich panels shear forces stiffness. Computation details are given in Appendix B. It leads to:

$$
\forall \underline{\boldsymbol{\gamma}}, \quad{ }^{t} \underline{\boldsymbol{\gamma}} \cdot \underset{\sim}{\boldsymbol{F}} \cdot \underline{\boldsymbol{\gamma}}<{ }^{t} \underline{\boldsymbol{\gamma}} \cdot \frac{\left\langle\underline{\boldsymbol{G}}^{c}\right\rangle_{c}}{k_{s}^{2}} \cdot \underline{\boldsymbol{\gamma}}
$$

where $\underset{\sim}{\boldsymbol{F}}$ is the actual shear forces stiffness tensor.

Here the effective transverse shear stiffness tensor of the core appears as: $G_{\alpha \beta}^{c \text {,eff }}=\left\langle\boldsymbol{G}^{c}\right\rangle_{c} / t_{c}$. It is the Voigt upper bound of transverse shear stiffness in 3D elasticity. Thus, we define the Voigt upper bound for shear forces stiffness as: $\underset{\sim}{\boldsymbol{F}} \mathrm{V}=\frac{\left\langle\boldsymbol{G}^{c}\right\rangle_{c}}{k_{s}^{2}}$.

It is also possible to derive a Reuss lower bound, assuming ad hoc compatible stress distribution in the skins and a uniform shear stress in the core. Computation details are given in Appendix B. This leads to the following Reuss bound:

$$
\forall \underline{\boldsymbol{\gamma}}, \quad{ }^{t} \boldsymbol{\boldsymbol { \gamma }} \cdot \frac{t_{c}^{2}}{k_{s}^{2}}\left(\left\langle\underset{\sim}{\left.\boldsymbol{g}^{c}\right\rangle_{c}}+l_{s} t_{s}{\underset{\sim}{\boldsymbol{g}}}^{s}\right)^{-1} \cdot \underline{\boldsymbol{\gamma}}<{ }^{t} \underline{\boldsymbol{\gamma}} \cdot \underset{\sim}{\boldsymbol{F}} \cdot \underline{\boldsymbol{\gamma}}\right.
$$

Again, the contribution of the skins does not vanish and the effective transverse shear stiffness of the core appears as: $G_{\alpha \beta}^{c, \text { eff }}=\left(\left\langle\underset{\sim}{\boldsymbol{g}^{c}}\right\rangle_{c} / t_{c}\right)^{-1}$. It is the Reuss lower bound for transverse shear stiffness in 3D elasticity. When the contribution of the skins is negligible, we define the Reuss lower bound for shear forces stiffness as: $\underset{\sim}{\boldsymbol{F}}=\frac{t_{c}^{2}}{k_{s}^{2}}\langle\underset{\sim}{\boldsymbol{g}}\rangle_{c}^{-1}$.

Since most of cores used in applications are porous, this bound is often null. In order to overcome this difficulty, Kelsey et al. [13] suggested improved bounds for sandwich panels.

\subsubsection{The bounds from Kelsey et al.}

The approach from Kelsey et al. [13] is also detailed in [44] and [32]. Let us recall that for the upper bound, a unit displacement is enforced only on the core, replacing the skins action on it (the unit displacement method). For the lower bound, it is an arbitrary stress distribution which is applied on the core (unit force method). It is possible to give partial justification to these bounds while separating the shear forces auxiliary problem $\mathcal{P}^{R M}$ in three parts, a part dedicated to the core and two parts dedicated to the skins. Then, the trial field in the skins is taken similar to the one used for Voigt and Reuss bounds in the previous section and the problem for the core is exactly the one suggested by Kelsey et al. [13].

For the upper bound, we still assume a uniform displacement in the the skins: $\underline{\hat{\boldsymbol{u}}}^{K+}=\frac{t_{c}}{2} \underline{\hat{\gamma}}$. Enforcing displacements continuity at the skin/core interface $\partial Y_{\text {int }}^{ \pm}$(Figure 1) leads to the following auxiliary problem in the core:

$$
\mathcal{P}^{K+}\left\{\begin{array}{l}
\hat{\boldsymbol{\sigma}}^{K+} \cdot \hat{\hat{\boldsymbol{\nabla}}}=0 \\
\stackrel{\hat{\boldsymbol{\sigma}}}{ }^{K+}=\hat{\boldsymbol{C}}(\underline{\hat{\boldsymbol{y}}}):\left(\hat{\boldsymbol{\nabla}} \otimes^{s} \underline{\hat{\boldsymbol{u}}}^{K+}\right) \\
\underline{\hat{\boldsymbol{u}}}^{K+}= \pm \frac{t_{c}}{2} \hat{\boldsymbol{\gamma}} \text { on interface faces } \partial Y_{i n t}^{ \pm} \\
\underline{\hat{\boldsymbol{\sigma}}}^{K+} \cdot \underline{\hat{\boldsymbol{n}}} \text { skew-periodic on } \partial Y_{l}^{c} \\
\underline{\hat{\boldsymbol{u}}}^{K+}\left(y_{1}, y_{2}, y_{3}\right) \quad\left(y_{1}, y_{2}\right) \text {-periodic on } \partial Y_{l}^{c}
\end{array}\right.
$$

which is exactly the unit displacement method suggested by Kelsey et al. [13]. Solving this problem for each components of $\underline{\gamma}$ leads to the following localization of the strain field in the core: $\varepsilon_{i j}^{K+}=e_{i j \alpha}^{K+} \gamma_{\alpha}$. The 
application of variational principles for the shear forces auxiliary problem $\mathcal{P}^{R M}$, detailed in Appendix B, leads to the following upper bound:

$$
\forall \underline{\boldsymbol{\gamma}}, \quad{ }^{t} \underline{\boldsymbol{\gamma}} \cdot \underset{\sim}{\boldsymbol{F}} \cdot \underline{\boldsymbol{\gamma}}<{ }^{t} \underline{\boldsymbol{\gamma}} \cdot{\underset{\sim}{\boldsymbol{F}}}^{K+} \cdot \underline{\boldsymbol{\gamma}}
$$

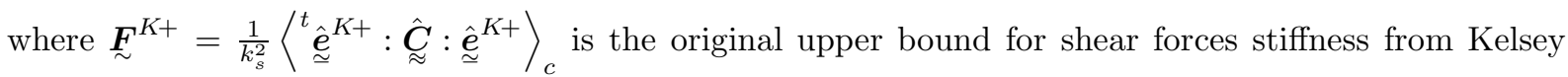
et al. [13].

The lower bound raises more difficulties. Separating the shear forces auxiliary problem (Problem 16) into three parts brings out the interface stress ${\underset{\boldsymbol{\sigma}}{(Q)}}^{(Q)}\left(y_{1}, y_{2}, \pm t_{c} / 2\right) \cdot\left( \pm \underline{\hat{\boldsymbol{e}}}_{3}\right)$ located on $\partial Y_{\text {int }}^{ \pm}$. This interfacial stress must respect macroscopic equilibrium for each part. Its resultant on each interface is:

$$
\frac{1}{A_{Y}} \int_{\partial Y_{i n t}^{ \pm}} \hat{\boldsymbol{\sigma}}^{(Q)}\left(y_{1}, y_{2}, \pm t_{c} / 2\right) \cdot \pm \underline{\hat{\boldsymbol{e}}}_{3} d y_{1} d y_{2}=\frac{1}{A_{Y}} \int_{Y^{s \pm}} \underline{\hat{\boldsymbol{f}}}^{(Q)} d y_{1} d y_{2} d y_{3}= \pm \frac{k_{s}}{t_{c}} \underline{\hat{\boldsymbol{Q}}}, \text { where } Q_{3}=0
$$

The derivation of the lower bound consists in choosing a priori this interfacial stress distribution. Let us introduce such arbitrary surface traction $\underline{\hat{\boldsymbol{T}}}^{d}\left(y_{1}, y_{2}\right)$ fulfilling:

$$
\frac{1}{A_{Y}} \int_{\partial Y_{i n t}^{+}} \underline{\hat{\boldsymbol{T}}}^{d} d \underline{\boldsymbol{y}}=\frac{k_{s}}{t_{c}} \hat{\boldsymbol{Q}}
$$

Given $\underline{\hat{\boldsymbol{T}}}^{d}\left(y_{1}, y_{2}\right)$, the problem in the core is stated as:

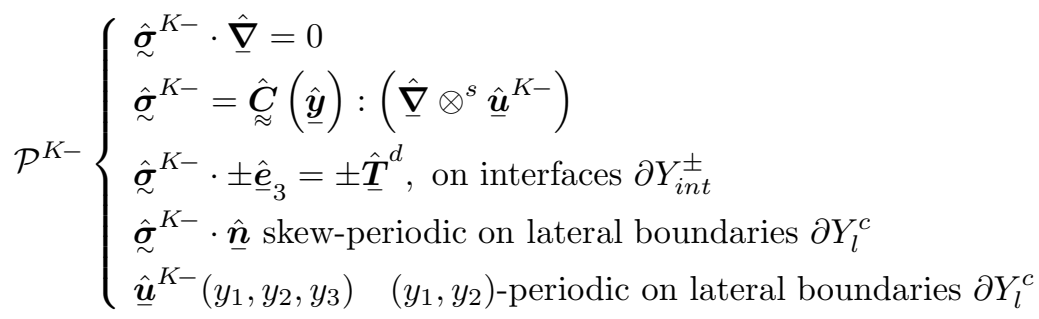

When $k_{s}=1$, this problem is exactly the one suggested by Kelsey et al. [13] for deriving the lower bound.

Then, the problems in the skins are stated as:

$$
\mathcal{P}^{K-, s \pm}\left\{\begin{array}{l}
\hat{\boldsymbol{\sigma}}^{K-} \cdot \hat{\hat{\boldsymbol{\nabla}}}+\frac{12 y_{3}}{h^{3}-t_{c}^{3}} \underline{\boldsymbol{Q}}=0 \\
\hat{\boldsymbol{\sigma}}^{K-}=\hat{\boldsymbol{C}}(\hat{\boldsymbol{y}}):\left(\hat{\boldsymbol{\boldsymbol { v }}} \otimes^{s} \underline{\boldsymbol{u}}^{K-}\right) \\
\hat{\boldsymbol{\sigma}}^{K-} \cdot \mp \underline{\hat{\boldsymbol{e}}}_{3}=\mp \underline{\boldsymbol{T}}^{d}, \text { on interface } \partial Y_{\text {int }}^{ \pm} \\
\hat{\boldsymbol{\sigma}}^{K-} \cdot \underline{\hat{\boldsymbol{e}}}_{3}=\hat{\mathbf{0}} \text { on free face } \partial Y_{3}^{ \pm} \\
\hat{\boldsymbol{\sigma}}^{K-} \cdot \underline{\hat{\boldsymbol{n}}} \text { skew-periodic on lateral boundaries } \partial Y_{l}^{s} \\
\underline{\hat{\boldsymbol{u}}}^{K-}\left(y_{1}, y_{2}, y_{3}\right) \quad\left(y_{1}, y_{2}\right) \text {-periodic on lateral boundaries } \partial Y_{l}^{s}
\end{array}\right.
$$

The combination of variational principles related to the three problems is detailed in Appendix B. This leads to the following lower bound for shear forces stiffness:

$$
\forall \underline{\boldsymbol{\gamma}}, \quad{ }^{t} \underline{\boldsymbol{\gamma}} \cdot\left({\underset{\sim}{\boldsymbol{f}}}^{K-}+{\underset{\sim}{\boldsymbol{f}}}^{K-, s+}+\underline{\sim}^{K-, s-}\right)^{-1} \cdot \underline{\boldsymbol{\gamma}}<^{t} \underline{\boldsymbol{\gamma}} \cdot \underset{\sim}{\boldsymbol{F}} \cdot \underline{\boldsymbol{\gamma}}
$$

where $\boldsymbol{f}^{K-}$ is the lower bound from Kelsey et al. [13] for the shear forces stiffness, and $\boldsymbol{f}^{K-, s \pm}$ are the contribution of the skins. 
The fact that the stress energy in the skins is negligible (even with thin skins) might be wrong in the general case. Yet this seems to be a very common implicit assumption. At best, one can hope that the skins contribution is of the same magnitude as the one in the case of the Reuss lower bound (Equation 17). In [33] a comparison between the full Bending-Gradient homogenization scheme and the bounds from Kelsey et al. [13] shows that the lower bound is not strictly respected.

\section{Discussion}

In Section 2, several questions were raised about the shear forces stiffness of sandwich panels. The assumptions usually made seemed antagonistic and the contribution of the skins was controversial. Additionally, there was a lack of well defined methods for deriving the shear forces stiffness when the sandwich panel is cellular. The application of the Bending-Gradient theory to sandwich panels enables us now to clarify these points.

First, the contrast assumption between the skins and the core was stated without explicit assumption about the skins thickness. When the core is homogeneous, Equation 12 is simply the generalization of the suggestion from Reissner [10] and compares plane-stress stiffnesses of the skins and the core, weighted by their respective thicknesses. With cellular cores, it is the plane part of the 3D elasticity stiffness of the core which must be compared to the plane-stress stiffness of the skins (Equation 14). If the constitutive materials of the skins and the core are isotropic and provided that Poisson's ratios are not close to 0.5 or -1 , the cellular case is almost identical to the homogeneous case.

Because the skins stiffness is weighted by their thickness, the contrast assumption might be rather restrictive. Actually its validity range can be enlarged in some cases. For example, this assumption applied to a cellular sandwich panel fully made of the same constitutive material leads to $\rho t_{c} \ll 2 t_{s}$ where $\rho$ is the volume fraction of material in the core. An approximate value for $\rho$ is $\rho \approx t_{f} / t_{c}$ where $t_{f}$ is the facets thickness of the walls constituting the core. This leads to $t_{f} \ll t_{s}$ which might seem very restrictive. However, depending on the actual contribution of the core to the flexural stiffness of the sandwich panel, this condition can be relaxed. For instance, folded cores contribution is almost negligible and $t_{f}$ can be almost comparable to $t_{s}$ without much violation of the contrast assumption [33]. This is not the case of honeycomb cores where the walls are vertical and contribute significantly to the flexural stiffness [28].

Furthermore, the contrast assumption does not state anything about the transverse shear stiffness of the skins. Hence $\boldsymbol{G}^{c} / t_{c} \ll \boldsymbol{G}^{s} / t_{s}$ (Equation 15) is usually valid but not necessary true a priori as illustrated with carbon fiber reinforced laminates. This remark leads us to the question of the contribution of the skins to the shear forces stiffness.

In order to avoid the difficulties regarding the contribution of the skins to the shear forces stiffness, it is commonly assumed that they are thin. In the present work, we did not invoke the thinness of the skins to derive the shear forces stiffness of any sandwich panel. It is possible to neglect the skins contribution afterward in order to retrieve classical results. As already pointed out this assumption goes against the contrast assumption and in many practical cases is not valid. This is especially the case with cellular cores where the skins are not fully glued to the core. One must keep the skins thick enough to prevent wrinkling.

Two contributions of the skins where identified. The first one, already included in the work from Kelsey et al. [13], is a pure geometric effect: the thicker the skins are, the further from the midplane the resultant of $\underline{\boldsymbol{f}}^{(Q)}$ in the skins is. Thus the lever effect of the resultant of $\underline{\boldsymbol{f}}^{(Q)}$ in the skins is greater (Figure 3 ). Taking the skins centroid as application point of $\underline{\boldsymbol{f}}^{(Q)}$ - as done in [4] - is only true at first order in $t_{s} / t_{c}$. This contribution is often not negligible and proportional to $1+2 t_{s} / t_{c}$. The second contribution of the skins was widely ignored. It involves directly the skins transverse shear stiffness. Even if, in the case of 
homogeneous core (Equation 15), it is rather easy to check that the effect is negligible, when the core is cellular this is much more difficult and uncertain.

In order to close discussion about the assumptions usually made with sandwich panels, let us explain why FOSDT is misleading and raised many questions [20]. When the shear correction factor is 1 , we have $\underset{\sim}{\boldsymbol{F}}=t_{c} \boldsymbol{G}^{c}+t_{s}{\underset{\sim}{\boldsymbol{G}}}^{s}$. Assuming simultaneously $\boldsymbol{G}^{c} \ll \boldsymbol{\sim}^{s}$ and $t_{s} \ll t_{c}$ leads to an undetermined limit. It is then not surprising that deriving shear correction factors following different methods leads to contradictions. Depending on the scaling between $\boldsymbol{G}^{c} \ll \boldsymbol{G}^{s}$ and $t_{s} \ll t_{c}$ and the order in which the assumptions are made, the correction might be completely different at limit cases. The sensitivity to limit case is illustrated on an isotropic sandwich panel in Appendix $\mathrm{C}$ where an exact formula is derived for shear forces stiffness. This situation illustrates clearly that it is much easier to use the concept of shear forces stiffness as a whole than to split it into uniform transverse shear and shear correction factor.

Finally, the main outcome of this work is the derivation and the justification of a direct homogenization scheme for sandwich panels shear forces stiffness. The contrast assumption enables not only to completely separate Kirchhoff-Love and shear force auxiliary problems, but also ensures that the constitutive equation is turned into a Reissner-Mindlin model whereas this is not the case for periodic plates in general ${ }^{3}$. The justification of already known bounds in the present work is a first validation of this new homogenization scheme. The shift from Bending-Gradient to Reissner-Mindlin models under contrast assumption presented in [33] is another validation and clearly illustrates the need of this new homogenization scheme when classical bounds are loose.

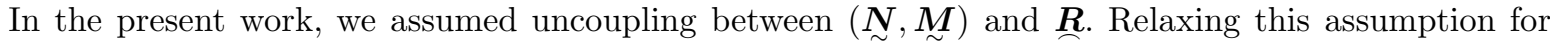
the core does not change the results regarding bounds and the direct homogenization scheme. However, the coupling will remain when deriving the constitutive equation (5) and it is not clear that one could neglect it in order to get a classical Reissner-Mindlin constitutive equation.

\section{Conclusion}

The application of the Bending-Gradient plate theory to a sandwich panel enabled us to give some justification of common results in sandwich theory. First, the application of the Kirchhoff-Love auxiliary problem brought out bounds for Kirchhoff-Love stiffnesses and a quantified contrast assumption. Then, we demonstrated that under this assumption, the shear constitutive equation for a sandwich panel is turned into a Reissner-Mindlin model as it is the case with homogeneous plates. Moreover, we identified the loading related to shear forces as a volume force distributed in the skins. This loading confirms the common intuition that the core is put into shear by the skins and enables the direct derivation of shear forces stiffness for a sandwich panel. Finally, we provided justification for bounds which are commonly used in sandwich panel design, taking into account the contribution of the skins.

\section{References}

[1] A. Lebée, K. Sab, A Bending-Gradient model for thick plates. Part I: Theory, International Journal of Solids and Structures 48 (2011) 2878-2888.

[2] V. S. Deshpande, N. A. Fleck, Collapse of truss core sandwich beams in 3-point bending, International Journal of Solids and Structures 38 (2001) 6275-6305.

3. For instance, it is demonstrated that the transverse shear behavior is dominated by warping effects with very common patterns in $[45]$ 
[3] K. Miura, Zeta-Core Sandwich - Its Concept and Realization, ISAS report 37 (1972) 137-164.

[4] H. Allen, Analysis and design of structural sandwich panels, Pergamon Press, 1969.

[5] A. K. Noor, W. S. Burton, C. W. Bert, Computational Models for Sandwich Panels and Shells, Applied Mechanics Reviews 49 (1996) 155-199.

[6] J. Hohe, W. Becker, Effective stress-strain relations for two-dimensional cellular sandwich cores: Homogenization, material models, and properties, Applied Mechanics Reviews 55 (2002) 61-87.

[7] E. Reissner, The effect of transverse shear deformation on the bending of elastic plates, Journal of Applied Mechanics 12 (1945) 68-77.

[8] R. Mindlin, Influence of rotatory Inertia and shear on flexural motions of isotropic, elastic plates, Journal of Applied Mechanics 18 (1951) 31-38.

[9] A. Lebée, Thick periodic plates homogenization, application to sandwich panels including chevron folded core, Ph.D. thesis, Université Paris-Est, 2010.

[10] E. Reissner, On bending of elastic plates, Quarterly of Applied Mathematics 5 (1947) 55 - 68.

[11] E. Reissner, Small Bending and Stretching of Sandwich-Type Shells, Tech. Rep. 1832, NACA, 1949.

[12] Hexcel, Honeycomb Sandwich Design Technology, Hexcel, 2000.

[13] S. Kelsey, R. A. Gellatly, B. W. Clark, The Shear Modulus of Foil Honeycomb Cores: A Theoretical and Experimental Investigation on Cores Used in Sandwich Construction, Aircraft Engineering and Aerospace Technology 30 (1958) $294-302$.

[14] J. N. Reddy, On refined computational models of composite laminates, International Journal for numerical methods in engineering 27 (1989) 361-382.

[15] H. Altenbach, Theories for laminated and sandwich plates. A review, Mechanics of Composite Materials 34 (1998) 243-252.

[16] A. K. Noor, M. Malik, An assessment of five modeling approaches for thermo-mechanical stress analysis of laminated composite panels, Computational Mechanics 25 (2000) 43-58.

[17] E. Carrera, Theories and finite elements for multilayered, anisotropic, composite plates and shells, Archives of Computational Methods in Engineering 9 (2002) 87-140.

[18] C. Libove, S. Batdorf, General small-deflection theory for flat sandwich plates, Tech. Rep., NACA, Washington, DC, United States, 1948.

[19] J. R. Vinson, Governing Equations for Plates and Panels of Sandwich Construction, in: Solid Mechanics and Its Applications, vol. 120, Springer Netherlands, 295-303, 2005.

[20] V. Birman, C. W. Bert, On the Choice of Shear Correction Factor in Sandwich Structures, Journal of Sandwich Structures and Materials 4 (2002) 83-95.

[21] G. Shi, P. Tong, The derivation of equivalent constitutive equations of honeycomb structures by a two scale method, Computational Mechanics 15 (1995) 395-407.

[22] X. F. Xu, P. Qiao, J. F. Davalos, Transverse Shear Stiffness of Composite Honeycomb Core with General Configuration, Journal of Engineering Mechanics 127 (2001) 1144-1151.

[23] J. Hohe, W. Becker, A mechanical model for two-dimensional cellular sandwich cores with general geometry, Computational Materials Science 19 (2000) 108 - 115.

[24] J. Hohe, W. Becker, An energetic homogenisation procedure for the elastic properties of general cellular sandwich cores, Composites Part B: Engineering 32 (2001) 185 - 197.

[25] J. Penzien, T. Didriksson, Effective Shear Modulus of Honeycomb Cellular Structure - Reply, Aiaa Journal 2 (1964) 1519-1519.

[26] M. Grediac, A Finite-Element Study Of The Transverse-Shear In Honeycomb-Cores, International Journal of Solids and Structures 30 (1993) 1777-1788.

[27] W. Becker, The in-plane stiffnesses of a honeycomb core including the thickness effect, Archive of Applied Mechanics 68 (1998) 334-341.

[28] A. Chen, J. F. Davalos, A solution including skin effect for stiffness and stress field of sandwich honeycomb core, International Journal of Solids and Structures 42 (2005) 2711 - 2739. 
[29] A. Chen, J. F. Davalos, Transverse Shear Including Skin Effect for Composite Sandwich with Honeycomb Sinusoidal Core, Journal of Engineering Mechanics 133 (2007) 247-256.

[30] G. Shi, P. Tong, Equivalent transverse shear stiffness of honeycomb cores, International Journal of Solids and Structures 32 (1995) 1383 - 1393.

[31] T. M. Nordstrand, L. A. Carlsson, Evaluation of transverse shear stiffness of structural core sandwich plates, Composite Structures 37 (1997) 145-153.

[32] A. Lebée, K. Sab, Transverse shear stiffness of a chevron folded core used in sandwich construction, International Journal of Solids and Structures 47 (2010) 2620-2629.

[33] A. Lebée, K. Sab, Homogenization of thick periodic plates: application of the Bending-Gradient plate theory to folded core sandwich panel, submitted (2011) .

[34] N. Buannic, P. Cartraud, T. Quesnel, Homogenization of corrugated core sandwich panels, Composite Structures 59 (2003) 299-312.

[35] J. Hohe, A direct homogenisation approach for determination of the stiffness matrix for microheterogeneous plates with application to sandwich panels, Composites Part B: Engineering 34 (2003) 615626.

[36] A. Cecchi, K. Sab, A homogenized Reissner-Mindlin model for orthotropic periodic plates: Application to brickwork panels, International Journal of Solids and Structures 44 (2007) 6055-6079.

[37] J. Whitney, Cylindrical Bending of Unsymmetrically Laminated Plates, Journal of Composite Materials 3 (1969) 715-719.

[38] A. Lebée, K. Sab, A Bending-Gradient model for thick plates, Part II: Closed-form solutions for cylindrical bending of laminates, International Journal of Solids and Structures 48 (2011) 2889-2901.

[39] J. M. Whitney, A. W. Leissa, Analysis of heterogeneous anisotropic plates, Journal of Applied Mechanics (1969) 261-266.

[40] D. Caillerie, Thin elastic and periodic plates., Mathematical Methods in the Applied Sciences 6 (1984) $159-191$.

[41] R. V. Kohn, M. Vogelius, A new model for thin plates with rapidly varying thickness, International Journal of Solids and Structures 20 (1984) 333-350.

[42] P. G. Ciarlet, P. Destuynder, Justification Of The 2-Dimensional Linear Plate Model, Journal de Mecanique 18 (1979) 315-344.

[43] A. G. Kolpakov, I. G. Sheremet, The stiffnesses of non-homogeneous plates, Journal of Applied Mathematics and Mechanics 63 (1999) 633-640.

[44] L. J. Gibson, M. F. Ashby, Cellular solids, Pergamon Press, 1988.

[45] A. Lebée, K. Sab, Homogenization of a space frame as a thick plate: application of the BendingGradient theory to a beam lattice, submitted (2011) .

[46] S. Vlachoutsis, Shear correction factors for plates and shells, Int. J. Numer. Meth. Engng. 33 (1992) $1537-1552$.

\section{Appendix A. Compatible fields for the Bending-Gradient theory}

Equilibrium equations and boundary conditions involving stress fields are gathered in the set of statically compatible fields: 


$$
\left\{\begin{array}{l}
\underset{\sim}{\boldsymbol{N}} \cdot \underline{\boldsymbol{\nabla}}=\underline{\mathbf{0}} \text { on } \omega \\
\underset{\sim}{\boldsymbol{M}} \otimes \underline{\boldsymbol{\nabla}}-\underline{\boldsymbol{R}}=\mathbf{0} \text { on } \omega \\
(\underset{\sim}{\boldsymbol{i}} \therefore \underline{\boldsymbol{R}}) \cdot \underline{\boldsymbol{\nabla}}=-p_{3} \text { on } \omega \\
\underset{\sim}{\boldsymbol{N}} \cdot \underline{\boldsymbol{n}}=\underline{\boldsymbol{V}}^{d} \text { on } \partial \omega^{s} \\
\underset{\sim}{\boldsymbol{M}}=\underline{\sim}^{d} \text { on } \partial \omega^{s} \\
(\underset{\sim}{\boldsymbol{i}} \therefore \underline{\boldsymbol{R}}) \cdot \underline{\boldsymbol{n}}=V_{3}^{d} \text { on } \partial \omega^{s}
\end{array}\right.
$$

where the derivation operator $\underline{\boldsymbol{\nabla}}$ is also formally represented as a vector: $\underset{\boldsymbol{a}}{\boldsymbol{\nabla}} \cdot \underline{\boldsymbol{\nabla}}=a_{\alpha \beta} \nabla_{\beta}=a_{\alpha \beta, \beta}$ is the divergence and $\underset{\sim}{\boldsymbol{a}} \otimes \underline{\boldsymbol{\nabla}}=a_{\alpha \beta} \nabla_{\gamma}=a_{\alpha \beta, \gamma}$ is the gradient. $\partial \omega^{s}$ is the portion of edge on which static boundary conditions apply and $\underline{\boldsymbol{n}}$ the related outer normal: $\hat{\boldsymbol{V}}^{d}=\left(V_{i}^{d}\right)$ is the force per unit length and $\boldsymbol{M}^{d}$ the full bending moment enforced on the edge. The plate is loaded exclusively with the out-of-plane distributed force $\underline{\hat{p}}=p_{3} \hat{\underline{e}}_{3}$. This set of equations is almost identical to Reissner-Mindlin equations where shear forces have been replaced by the bending gradient $\boldsymbol{R}$.

The compatibility conditions and boundary conditions for strain fields are gathered in the set of kinematically compatible fields:

$$
\left\{\begin{array}{l}
\underset{\sim}{\boldsymbol{e}}=\underset{\sim}{\boldsymbol{i}}:(\underline{\boldsymbol{\nabla}} \otimes \underline{\boldsymbol{U}}) \text { on } \omega \\
\underset{\boldsymbol{\chi}}{\boldsymbol{\Phi}} \cdot \underline{\boldsymbol{\Phi}} \cdot \underline{\boldsymbol{\nabla}} \text { on } \omega \\
\underset{\boldsymbol{\Gamma}}{\boldsymbol{\Phi}}+\underset{\sim}{\boldsymbol{i}} \cdot \underline{\boldsymbol{\nabla}} U_{3} \text { on } \omega \\
\underline{\boldsymbol{\Phi}} \cdot \underline{\boldsymbol{n}}=\underline{\sim}^{d} \text { on } \partial \omega^{k} \\
\hat{\hat{\boldsymbol{U}}}=\underline{\hat{\boldsymbol{U}}}^{d} \text { on } \partial \omega^{k}
\end{array}\right.
$$

where $\hat{\boldsymbol{U}}=\left(U_{i}\right)$ is the average through the thickness of the plate 3D displacement and $\underline{\boldsymbol{\Phi}}=\left(\Phi_{\alpha \beta \gamma}\right)$ is the generalized rotation. $\boldsymbol{\Gamma}$ and $\Phi$ are 2D-third-order tensors with the following symmetry: $\Phi_{\alpha \beta \gamma}=\Phi_{\beta \alpha \gamma}$. Moreover, $\partial \omega^{k}$ is the portion of edge on which kinematic boundary conditions apply: $\underline{\hat{\boldsymbol{U}}}^{d}=\left(U_{i}^{d}\right)$ is a given displacement and $\boldsymbol{H}^{d}=\left(H_{\alpha \beta}^{d}\right)$ is a symmetric second-order tensor related to a forced rotation on the edge. These fields are almost identical to Reissner-Mindlin kinematically compatible fields where the rotation vector is replaced by the generalized rotation $\underline{\Phi}$. Assuming $\Phi=\underset{\sim}{\boldsymbol{i}} \cdot \underline{\varphi}$ in (A.1), where $\underline{\varphi}$ is a vector representing rotations leads to Reissner-Mindlin-like kinematics: $\underset{\boldsymbol{\Gamma}}{\boldsymbol{i}} \underset{\sim}{\boldsymbol{i}} \cdot\left(\underline{\varphi}+\underline{\boldsymbol{\nabla}} U_{3}\right)=\underset{\sim}{\boldsymbol{i}} \cdot \underline{\boldsymbol{\gamma}}$ and $\underset{\sim}{\boldsymbol{\chi}}=\underset{\approx}{\boldsymbol{i}}:(\underline{\varphi} \otimes \underline{\nabla})$. Thus in the general case, Reissner-Mindlin kinematics can be interpreted as a restriction of $\Phi$ to $\underset{\sim}{\boldsymbol{i}} \cdot \underline{\varphi}$.

\section{Appendix B. Justification of bounds for the shear forces stiffness of sandwich panels}

In Section 5.3, is provided justification of bounds for the transverse shear stiffness of sandwich panels. Here the computation details are given. 
B.1. Upper bounds for sandwich panels shear forces stiffness

\section{B.1.1. Voigt upper bound}

We choose the following kinematically compatible strain field:

$$
\varepsilon_{\alpha 3}^{+}=\left\{\begin{array}{lc}
0 & \text { on } Y^{s \pm} \\
\frac{\gamma}{2} & \text { on } Y^{c}
\end{array} \quad \text { and } \quad \varepsilon_{\alpha \beta}^{+}=\varepsilon_{33}^{+}=0\right.
$$

$\varepsilon^{+}$enforces a uniform transverse shear strain in the core. $\varepsilon_{\sim}^{+}$is related to the following displacement field:

$$
\underline{\boldsymbol{u}}^{+}=\left\{\begin{array}{l} 
\pm \frac{t_{c}}{2} \underline{\boldsymbol{\gamma}} \text { on } Y^{s \pm} \\
\underline{\gamma} y_{3} \text { on } Y^{c}
\end{array} \quad \text { and } u_{3}^{+}=0\right.
$$

With this trial strain field $\varepsilon^{+}$the potential energy (11) of the Reissner-Mindlin shear auxiliary problem $\mathcal{P}^{R M}$ becomes:

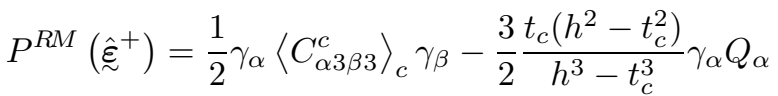

Minimizing $P^{R M}\left(\hat{\mathcal{\varepsilon}}^{+}\right)$with respect to $\underline{\gamma}$ leads to:

$$
\underline{\gamma}=k_{s}\left\langle\boldsymbol{G}^{c}\right\rangle_{c}^{-1} \cdot \underline{Q}
$$

Finally the potential energy for the Reissner-Mindlin auxiliary problem is:

$$
P^{R M}\left(\stackrel{\hat{\mathcal{\varepsilon}}}{+}^{+}\right)=-\frac{1}{2}^{t} \underline{\boldsymbol{Q}} \cdot k_{s}^{2}\left\langle\boldsymbol{G}^{c}\right\rangle_{c}^{-1} \cdot \underline{\boldsymbol{Q}}
$$

which leads to the Voigt upper bound:

$$
\forall \underline{Q}, \quad{ }^{t} \boldsymbol{Q} \cdot k_{s}^{2}\left\langle\underline{G}^{c}\right\rangle_{c}^{-1} \cdot \underline{\boldsymbol{Q}}<{ }^{t} \underline{\boldsymbol{Q}} \cdot \underset{\sim}{\boldsymbol{f}} \cdot \underline{\boldsymbol{Q}}
$$

\section{B.1.2. The upper bound from Kelsey et al.}

It is possible to give a sharper bound. We still assume a uniform displacement $\underline{\underline{\hat{u}}}^{K+}=\frac{t_{c}}{2} \hat{\underline{\gamma}}$ in the the skins and suggest the following auxiliary problem in the core:

$$
\mathcal{P}^{K+}\left\{\begin{array}{l}
\hat{\boldsymbol{\sigma}}^{K+} \cdot \underline{\hat{\boldsymbol{v}}}=0 \\
\hat{\boldsymbol{\sigma}}^{K+}=\underline{\hat{\boldsymbol{C}}}(\underline{\hat{\boldsymbol{y}}}):\left(\hat{\boldsymbol{\nabla}} \otimes^{s} \underline{\hat{\boldsymbol{u}}}^{K+}\right) \\
\underline{\hat{\boldsymbol{u}}}^{K+}= \pm \frac{t_{c}}{2} \underline{\boldsymbol{\gamma}} \text { on interface faces } \partial Y_{\text {int }}^{ \pm} \\
\hat{\boldsymbol{\sigma}}^{K+} \cdot \underline{\hat{\boldsymbol{n}}} \text { skew-periodic on } \partial Y_{l}^{c} \\
\underline{\hat{\boldsymbol{u}}}^{K+}\left(y_{1}, y_{2}, y_{3}\right) \quad\left(y_{1}, y_{2}\right) \text {-periodic on } \partial Y_{l}^{c}
\end{array}\right.
$$

This auxiliary problem is exactly the one suggested by Kelsey et al. [13] for deriving upper bounds of sandwich panels shear forces stiffness. The potential energy of this problem is:

$$
P^{K+}\left(\underline{\sim}^{K+}\right)=\frac{1}{2} \underline{\boldsymbol{\gamma}} \cdot\left\langle\underline{\underline{e}}^{t} \underline{\hat{e}}^{K+}: \underset{\approx}{\hat{C}}: \underline{\underline{e}}^{K+}\right\rangle_{c} \cdot \underline{\gamma}
$$

Then the potential energy of the core and the skins becomes:

$$
P^{R M}\left(\underline{\sim}^{\hat{\boldsymbol{\varepsilon}}^{K+}}\right)=\frac{1}{2} \underline{\boldsymbol{\gamma}}^{t} \cdot\left\langle\underline{\underline{\hat{e}}}^{K+}: \underset{\approx}{\hat{\boldsymbol{C}}}: \underline{\underline{\hat{e}}}^{K+}\right\rangle_{c} \cdot \underline{\boldsymbol{\gamma}}-k_{s} \underline{\boldsymbol{\gamma}} \cdot \underline{\boldsymbol{Q}}
$$


and the minimization over $\underline{\gamma}$ leads to:

$$
P^{R M}\left(\underline{\hat{\varepsilon}}^{K+}\right)=-\frac{1}{2} t \underline{\boldsymbol{Q}} \cdot k_{s}^{2}\left\langle\underline{\underline{e}}^{t}{ }^{K+}: \underset{\sim}{\hat{\boldsymbol{C}}}: \underline{\underline{e}}^{K+}\right\rangle_{c}^{-1} \cdot \underline{\boldsymbol{Q}}
$$

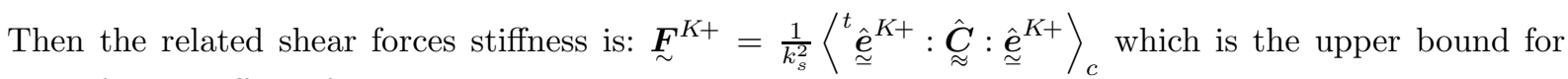
shear forces stiffness from Kelsey et al..

\section{B.2. Lower bound for sandwich panels shear forces stiffness}

\section{B.2.1. Reuss lower bound}

We choose a statically compatible stress field of the form:

$$
\sigma_{\alpha 3}^{-}=\left\{\begin{array}{l}
\frac{3}{2} \frac{h^{2}}{h^{3}-t_{c}^{3}}\left(1-\left(\frac{2 y_{3}}{h}\right)^{2}\right) Q_{\alpha} \text { on } Y^{s \pm} \\
\frac{3}{2} \frac{h^{2}-t_{c}^{2}}{h^{3}-t_{c}^{3}} Q_{\alpha} \quad \text { on } Y^{c}
\end{array} \quad \text { and } \sigma_{\alpha \beta}^{-}=\sigma_{33}^{-}=0\right.
$$

$\sigma^{-}$is uniform in the core and fulfills traction free boundary conditions on the upper and lower faces. With this definition of $\boldsymbol{\sigma}_{\sim}^{-}$, the complementary energy becomes:

$$
P^{* R M}\left(\hat{\boldsymbol{\sigma}}^{-}\right)=\frac{1}{2}^{t} \boldsymbol{Q} \cdot\left(\frac{k_{s}^{2}}{t_{c}^{2}}\left\langle{\underset{\sim}{\boldsymbol{g}}}^{c}\right\rangle_{c}+\frac{10 t_{c}^{2}+25 t_{c} t_{s}+16 t_{s}^{2}}{15\left(t_{c}+t_{s}\right)^{2}} t_{s} k_{s}^{2} \underline{\boldsymbol{g}}^{s}\right) \cdot \underline{\boldsymbol{Q}}
$$

\section{B.2.2. The lower bound from Kelsey et al.}

The complementary energy in the core derived with $\mathcal{P}^{K-}$ problem (Problem 18) is:

$$
P^{* K-}\left(\hat{\boldsymbol{\sigma}}^{K-}\right)=\frac{1}{2}\left\langle{ }^{t} \hat{\boldsymbol{\sigma}}^{K-}: \hat{\sim}^{c}(\underline{\hat{\boldsymbol{y}}}): \hat{\boldsymbol{\sigma}}^{K-}\right\rangle_{c}
$$

it is a quadratic form of $\boldsymbol{Q}$ which can be written as:

$$
P^{* K-}\left(\underline{\boldsymbol{\sigma}}^{K-}\right)=\frac{1}{2}{ }^{t} \boldsymbol{Q} \cdot \frac{k_{s}^{2}}{t_{c}} \boldsymbol{g}^{K-} \cdot \underline{\boldsymbol{Q}}
$$

Again, the complementary energy of problems in each skin $\mathcal{P}^{K-, s \pm}$ (Problem 19) is:

$$
P^{* K-, s \pm}\left(\hat{\boldsymbol{\sigma}}^{K-}\right)=\frac{1}{2}\left\langle{ }^{t} \hat{\boldsymbol{\sigma}}^{K-}: \hat{\boldsymbol{\sim}}^{c}(\underline{\hat{\boldsymbol{y}}}): \hat{\boldsymbol{\sigma}}^{K-}\right\rangle_{s \pm}
$$

it is a quadratic form of $\underline{Q}$ where it is possible to bring out the correction for thick skins:

$$
P^{* K-, s \pm}\left(\hat{\boldsymbol{\sigma}}^{K-}\right)=\frac{1}{2}^{t} \underline{\boldsymbol{Q}} \cdot \underline{\sim}^{K-, s \pm} \cdot \underline{\boldsymbol{Q}}
$$

Then the complementary energy of the whole unit-cell becomes:

$$
P^{* R M}\left({\underset{\sim}{\hat{\boldsymbol{q}}^{K-}}}^{K-}=\frac{1}{2}^{t} \underline{\boldsymbol{Q}} \cdot\left({\underset{\sim}{\boldsymbol{f}}}^{K-}+{\underset{\sim}{\boldsymbol{f}}}^{K-, s+}+{\underset{\sim}{\boldsymbol{f}}}^{K-, s-}\right) \cdot \underline{\boldsymbol{Q}}\right.
$$

where $\boldsymbol{f}_{\sim}^{K-}=\frac{k_{s}^{2}}{t_{c}} \boldsymbol{g}^{K-}$ is the lower bound for shear forces stiffness from Kelsey et al.. 


\section{Appendix C. An exact shear forces stiffness for isotropic sandwich panels}

We consider a sandwich panel made of isotropic materials having same Poisson's coefficient: ${\underset{\approx}{c}}^{c}=\epsilon \boldsymbol{\approx}^{s}$. Direct application of Equation 4 without any contrast assumption, reveals that in this specific case the transverse shear constitutive equation reduces to a Reissner-Mindlin model. Because the BendingGradient theory is closely related to the approach from Whitney [37] for deriving transverse shear stress distribution, similar results where derived in [46] and [20] but not interpreted this way. The corresponding Reissner-Mindlin shear forces stiffness writes as:

$$
\underset{\sim}{\boldsymbol{F}}=\frac{\left(2 \eta\left(4 \eta^{2}+6 \eta+3\right)+\epsilon\right)^{2}}{\epsilon^{2}+2 \epsilon \eta\left(5+5 \eta+10 \eta^{2}+25 \eta^{3}+16 \eta^{4}\right)+30 \eta^{2}(1+\eta)^{2}} \frac{5 t_{c}}{6} \boldsymbol{G}^{c}
$$

where $\eta=t_{s} / t_{c}$. It turns out that this formula is sensitive to the scaling between $\epsilon$ and $\eta$ when going to the limit. More precisely, assuming $\eta=0$ as well as $\eta=\infty$ leads to the shear forces stiffness of a homogeneous plate $(\underset{\sim}{\boldsymbol{F}}=5 / 6 h \boldsymbol{G})$. Assuming only stiffness contrast between skins and core $(\epsilon=0)$ leads to the formula from Kelsey et al. [13]. Furthermore assuming $\eta=0$ after $\epsilon=0$ lead to $\boldsymbol{F}=h \boldsymbol{G}^{c}$ which is different from the limit when only $\eta=0$.

More generally, setting a specific scaling between stiffness ratio and thickness ratio: $\epsilon=\eta^{\alpha}$ lead to different limit cases when $\eta$ goes to 0 . If $\alpha>1$ the skins stiffness increases fast enough to compensate thickness reduction and the limit case is $\underset{\sim}{\boldsymbol{F}}=h \underset{\sim}{\boldsymbol{G}^{c}}$. Whereas, if $\alpha<1$ the limit case is $\underset{\sim}{\boldsymbol{F}}=5 / 6 h \boldsymbol{\sim}^{c}$. Finally, $\alpha=1$ leads to $\underset{\sim}{\boldsymbol{F}}=245 / 246 h \boldsymbol{G}^{c}$. Beyond the game with limit cases, this observation illustrates the sensitivity to the order in which the assumptions are made by authors, when deriving shear forces stiffness for sandwich panels and explains some contradictions already pointed out in the literature. 